\title{
Products of degenerate quadratic forms
}

\author{
Paul Balmer
}

\begin{abstract}
We challenge the classical belief that products of degenerate quadratic forms must remain degenerate and we show that this fails in general, e.g. over tensor triangulated categories with duality. This opens new ways of constructing non-degenerate quadratic forms and hence classes in Witt groups. In addition, we encapsulate in a Leibniz-type formula the behavior of the product with respect to the symmetric cone construction. We illustrate these ideas by computing the total Witt group of regular projective spaces.
\end{abstract}

\section{Contents}

1 Triangulated categories defined over a topological space 1378

2 Symmetric forms, cones and Witt groups 1380

3 Support and degeneracy locus 1382

4 Product and consanguinity 1383

5 Leibniz formula $\quad 1385$

6 Pseudo-diagonal forms $\quad 1390$

7 Explicit examples over projective spaces $\quad 1394$

Appendix A. Recalling products, dualities and octahedra 1398

$\begin{array}{ll}\text { References } & 1404\end{array}$

\section{Introduction}

Perpetuation of degeneracy is the following well-known phenomenon. Given a degenerate symmetric form $\alpha_{1}$ on a finite-dimensional vector space $V_{1}$ and any symmetric form $\alpha_{2}$ on a space $V_{2}$, the tensor product symmetric form $\alpha_{1} \otimes \alpha_{2}$ on $V_{1} \otimes V_{2}$ is again degenerate, except, of course, in the trivial case where the space $V_{2}$ is zero, i.e. when $V_{1} \otimes V_{2}=0$. Perpetuation of degeneracy is not specific to vector spaces and holds similarly in all classical frameworks, such as for finitely generated projective modules over rings with involution or for vector bundles over schemes.

The present work builds on the surprising observation that perpetuation of degeneracy does not hold in more flexible frameworks, such as in triangulated categories with duality [Bal00, Bal01a]. To formalize this observation, we introduce a topological invariant of the forms $\alpha_{1}$ and $\alpha_{2}$, called the consanguinity of $\alpha_{1}$ and $\alpha_{2}$, which captures their inclination for a degenerate product. In particular, we prove the following.

Theorem. If $\alpha_{1}$ and $\alpha_{2}$ have no consanguinity then $\alpha_{1} \otimes \alpha_{2}$ is non-degenerate.

This is Corollary 4.6. The definition of consanguinity is given in $\S 4$.

Received 29 June 2004, accepted in final form 15 November 2004.

2000 Mathematics Subject Classification 11E81, 18E30, 19G12.

Keywords: product of symmetric forms, degeneracy, consanguinity, Witt groups.

Research supported by Swiss National Science Foundation, grant 620-66065.

This journal is (C) Foundation Compositio Mathematica 2005. 


\section{Products of Degenerate QuAdratic Forms}

We want to interpret the above theorem in terms of Witt groups, so let us briefly sketch the definition of triangular Witt groups. To do this, we need a notion introduced in [Bal00], namely the symmetric cone of a possibly degenerate symmetric form $\alpha$. This symmetric cone is a non-degenerate symmetric form associated to $\alpha$, that we denote by $d(\alpha)$ in the present paper. Symmetric spaces of the form $d(\alpha)$ are precisely the metabolic spaces and a symmetric form $\alpha$ is non-degenerate if and only if $d(\alpha)=0$. In particular $d(d(\alpha))=0$, which means that $d$ behaves as a differential: $d \circ d=0$. So, triangular Witt groups, which classify non-degenerate symmetric forms modulo metabolic ones, can be remembered as the homology of the complex defined by this symmetric cone construction $\alpha \mapsto d \alpha$. This is explained in $\S 2$. We have established in [Bal01a] that all classical Witt groups can be recovered as some triangular Witt groups, for suitable derived categories, at least if 2 is invertible in the original setting. The reader can find in the survey [Bal05] basic notions and motivations for the theory of classical and triangular Witt groups, in particular in algebraic geometry.

We now translate into Witt group language the appearance of non-degenerate symmetric forms as products of forms with no consanguinity. Indeed, this method allows us to construct Witt classes $\left[\alpha_{1} \otimes \alpha_{2}\right]$ out of two symmetric forms $\alpha_{1}$ and $\alpha_{2}$ which might be degenerate and hence might not define Witt classes themselves. In the form of a slogan, this reads

$$
\nexists\left[\alpha_{1}\right] \text { or } \nexists\left[\alpha_{2}\right] \text { but still } \exists\left[\alpha_{1} \otimes \alpha_{2}\right] .
$$

Before moving towards geometric applications, let us make a second general observation. Namely, assume that one of the forms, say $\alpha_{2}$, is metabolic, then it may happen that not only the product $\alpha_{1} \otimes \alpha_{2}$ is non-degenerate, as explained above, but is quite surprisingly non-metabolic. In some sense, the degeneracy of the form $\alpha_{1}$ can compensate the metabolicity of the form $\alpha_{2}$. Sloganized, this becomes

$$
\nexists\left[\alpha_{1}\right] \text { and }\left[\alpha_{2}\right]=0 \text { but }\left[\alpha_{1} \otimes \alpha_{2}\right] \neq 0 .
$$

These two observations (1) and (2) will be illustrated by geometric examples. They both imply that the Witt class of the product should not be understood as the product of the classes, at least in this generality: $\left[\alpha_{1} \otimes \alpha_{2}\right] \neq\left[\alpha_{1}\right] \cdot\left[\alpha_{2}\right]$.

In the presence of a differential $\alpha \mapsto d(\alpha)$ and of a product $\left(\alpha_{1}, \alpha_{2}\right) \mapsto \alpha_{1} \otimes \alpha_{2}$, it is legitimate to

wonder if these structures satisfy some type of Leibniz formula: $d\left(\alpha_{1} \otimes \alpha_{2}\right) \stackrel{?}{=} d\left(\alpha_{1}\right) \otimes \alpha_{2} \pm \alpha_{1} \otimes d\left(\alpha_{2}\right)$. For symmetric forms, this is not true in general because the left-hand side $d\left(\alpha_{1} \otimes \alpha_{2}\right)$ is always nondegenerate, as is $d(\alpha)$ for all $\alpha$, whereas the right-hand side is only conditionally non-degenerate. Here again, the consanguinity obstruction can be used for $d\left(\alpha_{1}\right)$ and $\alpha_{2}$, or, for $\alpha_{1}$ and $d\left(\alpha_{2}\right)$. Indeed, the consanguinity of $d\left(\alpha_{1}\right)$ and $\alpha_{2}$ is exactly equal to the consanguinity $\alpha_{1}$ and $d\left(\alpha_{2}\right)$ and coincides with the locus of common degeneracy of $\alpha_{1}$ and $\alpha_{2}$ (Proposition 5.1). When this obstruction is empty, we have the following.

Theorem (Leibniz-type formula). Let $\alpha$ and $\beta$ be symmetric forms whose degeneracy loci do not intersect. Then, we have an isometry

$$
d(\alpha \otimes \beta)=d(\alpha) \otimes \beta+\alpha \otimes d(\beta)
$$

up to signs which are made precise in $\S 5$.

This is Theorem 5.2, where + of course means the orthogonal sum. Unfortunately, the signs are not as easy as in the usual Leibniz formula.

We now want to see these abstract considerations at work in algebraic geometry.

In the last few years, triangular Witt theory [Bal00, Bal01a] led to a certain number of applications (see a survey in [Bal05]), among which the recent computation of the total Witt group 


\section{P. BALMER}

of projective bundles by Walter [Wal03], who considerably generalized Arason's famous theorem $\mathrm{W}\left(\mathbb{P}_{k}^{n}\right)=\mathrm{W}(k)$, see [Ara80], where $k$ was a field. The total Witt group of a scheme $X$

$$
\mathrm{W}^{\operatorname{Tot}}(X)=\bigoplus_{i \in \mathbb{Z} / 4} \bigoplus_{\mathcal{L} \in \operatorname{Pic}(X) / 2} \mathrm{~W}^{i}(X, \mathcal{L})
$$

is the graded ring of all (derived) Witt groups for all possible shifts $i$ and all possible twists $\mathcal{L}$ of the duality. Walter's computation constitutes a real tour de force, involving a precise description of derived categories of projective bundles. Over regular schemes though, this level of technicality is not always necessary and Witt groups can sometimes be computed by means of more geometric results such as Mayer-Vietoris, homotopy invariance and the like, as developed in [Bal01b]. Such a geometric strategy would typically consist in guessing the answer, in constructing a homomorphism globally, between this conjectured answer and the Witt groups under study, and in proving it an isomorphism locally. The crucial step, namely the construction of the custom-tailored global homomorphism, is where consanguinity might be used. We illustrate these ideas in $\S 7$ where we give a very simple geometric proof of Walter's theorem [Wal03] in the special but emblematic case of $\mathbb{P}_{X}^{n}$ with $X$ regular.

TheOREM. Let $X$ be a regular $\mathbb{Z}\left[\frac{1}{2}\right]$-scheme. Then the total graded Witt ring $\mathrm{W}^{\text {Tot }}\left(\mathbb{P}_{X}^{n}\right)$ is canonically a free $\mathrm{W}^{\text {Tot }}(X)$-module of rank 2 generated by the unit $\langle 1\rangle \in \mathrm{W}\left(\mathbb{P}_{X}^{n}\right)$ and one other class $\left[\beta_{X}^{(n)}\right] \in$ $\mathrm{W}^{n}\left(\mathbb{P}_{X}^{n}, \mathcal{O}(n+1)\right)$, whose square is zero.

This is Theorem 7.4 and we now explain how to construct the symmetric space $\beta_{X}^{(n)}$ defining the crucial generator $\left[\beta_{X}^{(n)}\right]$ by means of products of symmetric forms with no consanguinity. Indeed, our method is not specific to projective bundles and does not use regularity. (The regularity assumption comes from the geometric theorems of [Bal01b] mentioned above.) The general method goes as follows.

Let $X$ be a scheme. Consider $\mathcal{L} \in \operatorname{Pic}(X)$ a line bundle over $X$ and $s \in \Gamma(X, \mathcal{L})$ a global section. This can be seen as a one-dimensional 'diagonal' symmetric form $\alpha(s ; \mathcal{L})$ on the vector bundle $\mathcal{O}_{X}$ with respect to the $\mathcal{L}$-twisted (unshifted) duality

$$
\alpha(s ; \mathcal{L}):=\left(\mathcal{O}_{X}, \mathcal{O}_{X} \stackrel{s}{\longrightarrow} \mathcal{L}\right) .
$$

Such a symmetric form $\alpha(s ; \mathcal{L})$ is usually degenerate, unless $s$ yields a trivialization of the line bundle $\mathcal{L}$. The product of a finite number of such symmetric forms $\alpha\left(s_{1} ; \mathcal{L}_{1}\right) \otimes \cdots \otimes \alpha\left(s_{m} ; \mathcal{L}_{m}\right)$ $=\alpha\left(s_{1} \otimes \cdots \otimes s_{m} ; \mathcal{L}_{1} \otimes \cdots \otimes \mathcal{L}_{m}\right)$ still has the same nature and degeneracy clearly tends to increase in this process. So, this cannot lead us to a non-degenerate symmetric space unless we are simply considering a good old one-dimensional form $\langle u\rangle$ for a global unit $u \in \Gamma\left(X, \mathcal{O}_{X}\right)^{\times}$, and this would not be worth the trouble. However, we can also consider mixed products involving one diagonal form $\alpha\left(s_{0} ; \mathcal{L}_{0}\right)$ as well as symmetric cones $d \alpha\left(s_{i} ; \mathcal{L}_{i}\right)$ for $i=1, \ldots, n$. Orthogonal sums of such mixed products are the pseudo-diagonal forms of $\S 6$. Pseudo-diagonal forms may be non-degenerate without necessarily being mere diagonal forms $\left\langle u_{1}, \ldots, u_{n}\right\rangle$ for global units $u_{1}, \ldots, u_{n}$. Indeed, using consanguinity methods, we establish in Corollary 6.13 the following result.

Theorem. Let $n \geqslant 0$, let $\mathcal{L}_{0}, \ldots, \mathcal{L}_{n}$ be $n+1$ line bundles over a scheme $X$ and let $s_{i} \in \Gamma\left(X, \mathcal{L}_{i}\right)$, for $i=0, \ldots, n$, be global sections which do not vanish simultaneously: $\bigcap_{i=0}^{n} Z\left(s_{i}\right)=\varnothing$. Then

$$
\alpha\left(s_{0} ; \mathcal{L}_{0}\right) \otimes \mathbf{d} \alpha\left(s_{1} ; \mathcal{L}_{1}\right) \otimes \cdots \otimes \mathbf{d} \alpha\left(s_{n} ; \mathcal{L}_{n}\right)
$$

is a non-degenerate symmetric space and defines a class in the Witt group $\mathrm{W}^{n}(X, \mathcal{L})$ where $\mathcal{L}=$ $\mathcal{L}_{0} \otimes \cdots \otimes \mathcal{L}_{n}$. 


\section{Products of DEGENERATE QUADRATIC FORMS}

It is an interesting open question to know for which schemes the total Witt group is generated by such pseudo-diagonal spaces. This would be a global version of the well-known diagonalization theorems over fields and local rings.

In any case, if we apply this to the scheme $\mathbb{P}_{X}^{n}$, to $\mathcal{L}_{0}=\cdots=\mathcal{L}_{n}=\mathcal{O}(1)$ and to $s_{i}=T_{i}$ (the homogeneous coordinates), then it is clear that $\bigcap_{i=0}^{n}\left\{T_{i}=0\right\}=\varnothing$ and that the above result provides us with a non-degenerate space. This is nothing but the announced generator of the total Witt group of $\mathbb{P}_{X}^{n}$ :

$$
\beta_{X}^{(n)}=\alpha\left(T_{0} ; \mathcal{O}(1)\right) \otimes d \alpha\left(T_{1} ; \mathcal{O}(1)\right) \otimes \cdots \otimes d \alpha\left(T_{n} ; \mathcal{O}(1)\right)
$$

This quite non-trivial application illustrates the strength of the abstract machinery of consanguinity. It also provides examples of the above 'surprises' (1) and (2). Let us also stress that consanguinity does not need to be applied only to diagonal forms but is a very general concept.

Although slightly beside the point of this article, let us briefly comment on the various projective bundle theorems for Witt groups. This is easy to summarize: only Walter [Wal03] reaches maximal generality. Note that for non-trivial projective bundles $\mathbb{P}(\mathcal{E})$, Walter's description is not always as simple as above and can involve a non-split exact sequence of Witt groups. As already mentioned, Walter does not use regularity of the ground scheme. For the history between Arason and Walter, we refer to [Wal03]. For the very recent post-Walter times, let us mention Nenashev's current series of articles (see [Nen04] and more references therein), which also provide a geometric approach to Witt groups of projective bundles over a regular basis, using non-oriented cohomology theories and deformation to the normal cone techniques. Note that Nenashev also considers $\mathbb{P}(\mathcal{E})$ for some vector bundles $\mathcal{E}$.

However, the goal of the present article is certainly not the projective bundle theorem itself. This only appears as a nice by-product of our main theme: the study of non-degenerate products of possibly degenerate symmetric forms.

Let us briefly review the part of the material not mentioned so far.

Our natural language is that of algebraic geometry, that is, the reader could have in mind his favorite scheme $X$ and various derived categories over $X$. Although everything could be expressed at this level of generality, we introduce a more abstract language, namely that of a triangulated category defined over a topological space $X$, see $\S 1$. This has the following advantages. First, even in the above algebro-geometric examples, it will avoid making a different story for each type of derived category we can associate to $X$ (of vector bundles, of coherent modules, of perfect complexes, etc.) and it also makes clear which geometric properties are really needed. Moreover, of course, our general formalism can possibly serve outside this algebro-geometric context.

The short $\S \S 2$ and 3 contain basic notions about symmetric forms, such as degeneracy, support, symmetric cones, Witt groups and the like. Although not revolutionary, the presentation of Witt groups as the homology groups of the graded semiring of possibly degenerate symmetric forms, see Proposition 2.5, ideally prepares the reader's mind for the Leibniz formula of $\S 5$.

Section 4 deals with products and consanguinity. We essentially use, in triangular Witt theory, the product of Gille and Nenashev [GN03], except for the simplifying trick they introduced in their main definition, a trick which only works if the forms are non-degenerate, that is precisely what we cannot afford to assume here. We use instead a more natural construction, but in the same framework, namely that of 'triangulated categories with product and duality' or TPD-categories, recalled in Appendix A for the reader's convenience. In order to prove the above Leibniz formula in $\S 5$, we also need to control the behaviour of the tensor product with respect to the triangulation in a more precise way than just requiring the product be exact in each variable. For this, we need May's recent axiomatization [May01], revamped à la Keller-Neeman [KN02] and also transcripted in Appendix A. 


\section{P. BALMER}

\section{Preliminaries and conventions}

Remark 0.1. In mathematics, some things must be made explicit, some other things must absolutely not. This circumspection applies, in particular, to natural isomorphisms. Here, our rule is to label those natural isomorphisms which are relevant to the current argument and to consider as identities those which are not.

Convention 0.2. We assume without mention that 2 is invertible, i.e. our schemes are $\mathbb{Z}\left[\frac{1}{2}\right]$-schemes and our categories with duality are $\mathbb{Z}\left[\frac{1}{2}\right]$-categories.

Convention 0.3. A scheme is called regular if it is noetherian, separated and locally regular.

Notation 0.4. Grothendieck-Verdier's notion of triangulated category is defined in [Ver96]. We usually assume that our triangulated categories are essentially small. We denote by $T: \mathcal{K} \longrightarrow \mathcal{K}$ the translation functor in the triangulated category $\mathcal{K}$ (also known as the 'suspension' in topology or the 'shift' in homological algebra).

Definition 0.5. Let $u: A \rightarrow B$ be a morphism in a triangulated category. We call the cone of $u$ any object $C$, or more precisely any triple $\left(C, u_{1}, u_{2}\right)$, such that the triangle $A \stackrel{u}{\longrightarrow} B \stackrel{u_{1}}{\longrightarrow} C \stackrel{u_{2}}{\longrightarrow} T A$ is distinguished. For a fixed morphism $u$, its cone is unique up to non-unique isomorphism and we denote it by cone $(u)$.

\section{Triangulated categories defined over a topological space}

Definition 1.1. Let $X$ be a topological space. A triangulated category defined over $X$ is a pair $(\mathcal{K}$, supp) where $\mathcal{K}$ is a triangulated category and supp assigns to each object $A \in \mathcal{K}$ a closed subset of $X$

$$
\operatorname{supp}(A) \subset X
$$

called the support of $A$ and subject to the following four elementary rules.

(S1) Only the support of zero is $\operatorname{empty:} \operatorname{supp}(A)=\varnothing \Leftrightarrow A \cong 0$.

(S2) The support respects direct sums: $\operatorname{supp}(A \oplus B)=\operatorname{supp}(A) \cup \operatorname{supp}(B)$.

(S3) The support respects translation: $\operatorname{supp}(A)=\operatorname{supp}(T A)$.

(S4) The support respects distinguished triangles: if $A \longrightarrow B \longrightarrow C \longrightarrow T A$ is a distinguished triangle then $\operatorname{supp}(C) \subset \operatorname{supp}(A) \cup \operatorname{supp}(B)$.

Remark 1.2. By the rotation axiom and by (S3), we can equivalently say in $(\mathrm{S} 4)$ that $\operatorname{supp}(A) \subset$ $\operatorname{supp}(B) \cup \operatorname{supp}(C)$ or that $\operatorname{supp}(B) \subset \operatorname{supp}(A) \cup \operatorname{supp}(C)$. It follows from this and from (S1) that the support respects isomorphisms $A \simeq B \Rightarrow \operatorname{supp}(A)=\operatorname{supp}(B)$.

Example 1.3. Let $X$ be a scheme. The following are triangulated categories over the underlying topological space of $X$. In all cases below, the support is the homological support, that is, the usual support of the total homology $\mathcal{O}_{X}$-module.

(a) Let $\mathcal{K}=\mathbb{D}^{\mathrm{b}}\left(\mathrm{VB}_{X}\right)$ be the derived category of bounded complexes of vector bundles over $X$. (We use $\mathbb{D}$ so as not to confuse with the $D$ of dualities below.)

(b) Assume that $X$ is noetherian and let $\mathcal{K}=\mathbb{D}^{\mathrm{b}}\left(\mathrm{Coh}_{X}\right)$ be the derived category of bounded complexes of coherent $\mathcal{O}_{X}$-modules.

(c) Let $\mathcal{K}=\mathbb{D}^{\text {perf }}(X)$ be the derived category of perfect complexes over $X$.

In all derived categories, for shifts and mapping cones, we follow the (homological) sign conventions of Weibel [Wei94]. 


\section{Products OF DEGENERATE QUADRATIC FORMS}

Definition 1.4. Let $(\mathcal{K}$, supp) be a triangulated category defined over $X$ as in Definition 1.1. Assume that $\mathcal{K}$ carries a structure $(\mathcal{K}, D, \delta, \varpi)$ of triangulated category with duality as recalled in Definition A.2. We say that $\mathcal{K}$ is a triangulated category with duality defined over $X$ if:

(S5) the support respects the duality: $\operatorname{supp}(D A)=\operatorname{supp}(A)$.

Definition 1.5. Let $\left(\mathcal{K}, \operatorname{supp}_{\mathcal{K}}\right),\left(\mathcal{L}, \operatorname{supp}_{\mathcal{L}}\right)$ and $\left(\mathcal{M}, \operatorname{supp}_{\mathcal{M}}\right)$ be triangulated categories defined over $X$ as in Definition 1.1. Assume that $\square: \mathcal{K} \times \mathcal{L} \rightarrow \mathcal{M}$ is a pairing of triangulated categories as recalled in Definition A.1. We say that the pairing $\otimes$ is defined over $X$ if:

(S6) the support respects the product: $\operatorname{supp}_{\mathcal{M}}(A \otimes B)=\operatorname{supp}_{\mathcal{K}}(A) \cap \operatorname{supp}_{\mathcal{L}}(B)$.

Indeed, in most of what follows, we only use the inclusion $\operatorname{supp}_{\mathcal{M}}(A \otimes B) \subset \operatorname{supp}_{\mathcal{K}}(A) \cap \operatorname{supp}_{\mathcal{L}}(B)$. In the interesting examples, especially the geometric examples, equality does hold.

Definition 1.6. A pairing of triangulated categories with duality defined over $X$ is the data of three triangulated categories $\mathcal{K}, \mathcal{L}$ and $\mathcal{M}$, all equipped with a duality, all defined over $X$, and of a pairing $\nabla: \mathcal{K} \times \mathcal{L} \rightarrow \mathcal{M}$ of triangulated categories with duality in the sense of [GN03] (see Definition A.4), such that both axioms (S5) and (S6) are satisfied.

In the special case where $\mathcal{K}=\mathcal{L}=\mathcal{M}$, we say that $\mathcal{K}$ is a TPD-category defined over $X$, or longer: a triangulated category with product and duality, defined over $X$.

Example 1.7. In Example 1.3(a) and (c), the categories are equipped with product and duality without further assumptions, by simply deriving the usual ones on vector bundles. They define TPD-categories over $X$ in the sense of Definition 1.6. We shall also consider dualities twisted by line bundles, as usual. In Example 1.3(b), it is recommended to assume $X$ be Gorenstein of finite Krull dimension to get a duality (see [Gil02]). The author does not know of a good condition for the existence of a reasonable tensor product on $\mathbb{D}^{\mathrm{b}}\left(\mathrm{Coh}_{X}\right)$ itself. More common is the pairing $\mathbb{D}^{\mathrm{b}}\left(\mathrm{VB}_{X}\right) \times \mathbb{D}^{\mathrm{b}}\left(\mathrm{Coh}_{X}\right) \rightarrow \mathbb{D}^{\mathrm{b}}\left(\mathrm{Coh}_{X}\right)$ which is defined over $X$ in the above sense. This external pairing turns $\mathbb{D}^{\mathrm{b}}\left(\mathrm{Coh}_{X}\right)$ into a module over $\mathbb{D}^{\mathrm{b}}\left(\mathrm{VB}_{X}\right)$ and illustrates why external pairings are really necessary.

Remark 1.8. It is clear from Proposition A.7 and from axiom (S3) that shifting the dualities on $\mathcal{K}, \mathcal{L}$ and coherently on $\mathcal{M}$ still produces a pairing of triangulated categories with duality defined over $X$.

Remark 1.9. A naive misconception would be to think that the cone of a morphism of the form $u \otimes u^{\prime}$ simply is the product $\otimes$ of the cones of $u$ and $u^{\prime}$. The situation is more complicated, as explained in Appendix A. The following statement gives some control on supports of such cones.

Lemma 1.10. Let $\nabla: \mathcal{K} \times \mathcal{L} \longrightarrow \mathcal{M}$ be a pairing of triangulated categories over $X$. Consider two morphisms $u: A \rightarrow B$ in $\mathcal{K}$ and $u^{\prime}: A^{\prime} \rightarrow B^{\prime}$ in $\mathcal{L}$. Let us denote their cones by $C:=\operatorname{cone}(u) \in \mathcal{K}$ and $C^{\prime}:=\operatorname{cone}\left(u^{\prime}\right) \in \mathcal{L}$. Then the support of the cone of $u \otimes u^{\prime}$ is contained in the following four-term union:

$$
\begin{aligned}
\operatorname{supp}_{\mathcal{M}}\left(\operatorname{cone}\left(u \otimes u^{\prime}\right)\right) & \subset \operatorname{supp}_{\mathcal{K}}(A) \cap \operatorname{supp}_{\mathcal{K}}(B) \cap \operatorname{supp}_{\mathcal{L}}\left(C^{\prime}\right) \\
& \cup \operatorname{supp}_{\mathcal{L}}\left(A^{\prime}\right) \cap \operatorname{supp}_{\mathcal{L}}\left(B^{\prime}\right) \cap \operatorname{supp}_{\mathcal{K}}(C) \\
& \cup \operatorname{supp}_{\mathcal{K}}(A) \cap \operatorname{supp}_{\mathcal{L}}\left(A^{\prime}\right) \cap \operatorname{supp}_{\mathcal{K}}(C) \cap \operatorname{supp}_{\mathcal{L}}\left(C^{\prime}\right) \\
& \cup \operatorname{supp}_{\mathcal{K}}(B) \cap \operatorname{supp}_{\mathcal{L}}\left(B^{\prime}\right) \cap \operatorname{supp}_{\mathcal{K}}(C) \cap \operatorname{supp}_{\mathcal{K}}\left(C^{\prime}\right) .
\end{aligned}
$$

If, moreover, $\otimes$ is a pairing of triangulated categories with duality over $X$ and if $B=D_{\mathcal{K}} A$ and $B^{\prime}=D_{\mathcal{L}} A^{\prime}$, then the above reduces to

$$
\operatorname{supp}_{\mathcal{M}}\left(\operatorname{cone}\left(u \otimes u^{\prime}\right)\right) \subset \operatorname{supp}_{\mathcal{K}}(A) \cap \operatorname{supp}_{\mathcal{L}}\left(C^{\prime}\right) \cup \operatorname{supp}_{\mathcal{L}}\left(A^{\prime}\right) \cap \operatorname{supp}_{\mathcal{K}}(C) .
$$




\section{P. BALMER}

Proof. Let us abbreviate $C^{\prime \prime}:=\operatorname{cone}\left(u \otimes u^{\prime}\right) \in \mathcal{M}$. We have by definition two distinguished triangles in $\mathcal{K}$ and $\mathcal{L}$ as follows:

$$
A \stackrel{u}{\longrightarrow} B \longrightarrow C \longrightarrow T A \text { and } A^{\prime} \stackrel{u^{\prime}}{\longrightarrow} B^{\prime} \longrightarrow C^{\prime} \longrightarrow T\left(A^{\prime}\right) .
$$

From the relation

$$
u \otimes u^{\prime}=\left(u \otimes \operatorname{id}_{B^{\prime}}\right) \circ\left(\operatorname{id}_{A} \otimes u^{\prime}\right),
$$

the octahedron axiom guarantees the existence of a distinguished triangle relating the cones of these three morphisms. The cone of $u \nabla u^{\prime}$ is our object $C^{\prime \prime}$ by definition and the cones of $u \nabla \operatorname{id}_{B^{\prime}}$ and $\operatorname{id}_{A} \otimes u^{\prime}$ are simply obtained from the above distinguished triangles by applying the exact functors $-\otimes B^{\prime}$ and $A \otimes-$, respectively. So, we have from the octahedron a distinguished triangle as follows:

$$
A \otimes C^{\prime} \longrightarrow C^{\prime \prime} \longrightarrow C \otimes B^{\prime} \longrightarrow T\left(A \otimes C^{\prime}\right) .
$$

By axiom $(\mathrm{S} 4)$, we deduce $\operatorname{supp}_{\mathcal{M}}\left(C^{\prime \prime}\right) \subset \operatorname{supp}_{\mathcal{M}}\left(A \otimes C^{\prime}\right) \cup \operatorname{supp}_{\mathcal{M}}\left(C \otimes B^{\prime}\right) \stackrel{\left(\mathrm{S}^{\prime}\right)}{=} \operatorname{supp}_{\mathcal{K}}(A) \cap$ $\operatorname{supp}_{\mathcal{L}}\left(C^{\prime}\right) \cup \operatorname{supp}_{\mathcal{K}}(C) \cap \operatorname{supp}_{\mathcal{L}}\left(B^{\prime}\right)$. Using the other decomposition $u \otimes u^{\prime}=\left(\operatorname{id}_{B} \otimes u^{\prime}\right) \circ\left(u \nabla \operatorname{id}_{A^{\prime}}\right)$, we establish similarly the inclusion $\operatorname{supp}_{\mathcal{M}}\left(C^{\prime \prime}\right) \subset \operatorname{supp}_{\mathcal{K}}(B) \cap \operatorname{supp}_{\mathcal{L}}\left(C^{\prime}\right) \cup \operatorname{supp}_{\mathcal{K}}(C) \cap \operatorname{supp}_{\mathcal{L}}\left(A^{\prime}\right)$. Hence, $\operatorname{supp}_{\mathcal{M}}\left(C^{\prime \prime}\right)$ is contained in the intersection of the two sets we just found, which gives the set of the statement.

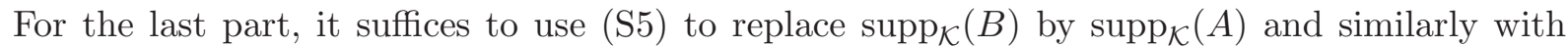
$B^{\prime}$ and $A^{\prime}$. In this case, the above four-term union boils down to the announced one, as is easily checked.

\section{Symmetric forms, cones and Witt groups}

For this section, $(\mathcal{K}, D, \delta, \varpi)$ is a triangulated category with duality as recalled in Definition A.2. Although quite standard, we fix the following terminology since the distinction between degenerate and non-degenerate forms is essential here.

Definition 2.1. We define a symmetric pair to be a couple $(A, a)$ formed by an object $A \in \mathcal{K}$ and a symmetric morphism $a: A \rightarrow D A$. As usual, symmetry means $D(a) \circ \varpi_{A}=a$. Note that we do not require $a$ to be an isomorphism. The morphism $a$ is referred to as the form of the symmetric pair $(A, a)$.

When $a$ is, moreover, an isomorphism, we say that the form $a: A \rightarrow D A$ is non-degenerate and that the symmetric pair $(A, a)$ is a symmetric space.

Let $i \in \mathbb{Z}$ be an integer. A symmetric $i$-pair, a symmetric $i$-space, an $i$-form, with respect to the duality $D$, respectively mean a symmetric pair, a symmetric space, a form for the $i$ th shifted duality $\left(D^{(i)}, \delta^{(i)}, \varpi^{(i)}\right)$ over $\mathcal{K}$, as recalled in Definition A.3.

Notations 2.2. We define isometries of symmetric pairs as usual and denote by

$$
\operatorname{Symm}(\mathcal{K}) \quad \text { or } \operatorname{Symm}(\mathcal{K}, D, \delta, \varpi)
$$

the monoid of isometry classes of symmetric forms over the considered category with duality. Our assumption about essential smallness of $\mathcal{K}$ implies that $\operatorname{Symm}(\mathcal{K})$ is a set. It is a monoid with the usual orthogonal sum. We shall not adopt a new notation for the class of a symmetric pair $(A, a)$ in $\operatorname{Symm}(\mathcal{K})$ and simply write it as $(A, a)$. For an integer $i \in \mathbb{Z}$, we denote by

$$
\operatorname{Symm}^{(i)}(\mathcal{K}):=\operatorname{Symm}\left(\mathcal{K}, D^{(i)}, \delta^{(i)}, \varpi^{(i)}\right)
$$

the corresponding monoid for the $i$ th shifted duality.

Definition 2.3. We now recall from $[$ Bal00, $\S 2]$ the notion of symmetric cone of a symmetric form. The cone of a morphism $a: A \rightarrow B$ is recalled in Definition 0.5. If $B=D A$ and if the morphism $a$ is symmetric, then its cone $C=\operatorname{cone}(a)$ also becomes symmetric, in the sense that 


\section{Products of Degenerate QuAdratic Forms}

it carries a non-degenerate symmetric form, but for the 1-shifted duality $D^{(1)}$. Namely there exists a symmetric 1-space $(C, \Phi)$, unique up to isometry [Bal00, Theorem 2.6], such that the following triangle is distinguished:

$$
A \stackrel{a}{\longrightarrow} D A \stackrel{a_{1}}{\longrightarrow} C \stackrel{a_{2}}{\longrightarrow} T A
$$

and is symmetric in the sense that the following equation holds

$$
\Phi \circ a_{1}=-T\left(D\left(a_{2}\right)\right) .
$$

It is equivalent to say that $(C, \Phi)$ is a 1 -space such that the following diagram with distinguished rows commutes:

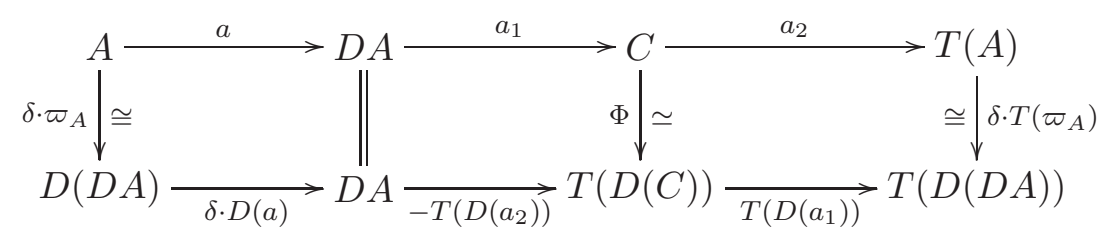

in which the second row is the dual of the first.

The symmetry of $\Phi: C \longrightarrow D^{(1)}(C)$ for the 1 -shifted duality $D^{(1)}=T \circ D$ reads by definition: $D^{(1)}(\Phi) \circ \varpi_{C}^{(1)}=\Phi$. Unfolding Definition A.3 gives $-\delta \cdot T(D(\Phi)) \circ \varpi_{C}=\Phi$. This new symmetric space is denoted by

$$
\operatorname{Cone}(A, a):=(C, \Phi)
$$

and is the symmetric cone of the symmetric pair $(A, a)$. See example 3.5.

Remark 2.4. In the next statement, we use freely the language of triangular Witt groups (TWG) $[$ Bal00, § 2]. The reader unfamiliar with TWG can consider the following as (quite conceptual) definitions for the monoids $\mathrm{MW}^{i}(\mathcal{K}), \mathrm{NW}^{i}(\mathcal{K})$ and the groups $\mathrm{W}^{i}(\mathcal{K})$.

Proposition 2.5. Let $(\mathcal{K}, D, \delta, \varpi)$ be a triangulated category with duality. The symmetric cone construction (see Definition 2.3) induces for all $i \in \mathbb{Z}$ a well-defined homomorphism of monoids:

$$
\begin{aligned}
d: \operatorname{Symm}^{(i)}(\mathcal{K}) & \longrightarrow \operatorname{Symm}^{(i+1)}(\mathcal{K}) \\
(A, a) & \longmapsto \operatorname{Cone}(A, a)
\end{aligned}
$$

which enjoys the following properties.

(a) The homomorphism $d$ is a differential: $d \circ d=0$.

$$
\ldots \stackrel{d}{\longrightarrow} \operatorname{Symm}^{(i-1)}(\mathcal{K}) \stackrel{d}{\longrightarrow} \operatorname{Symm}^{(i)}(\mathcal{K}) \stackrel{d}{\longrightarrow} \operatorname{Symm}^{(i+1)}(\mathcal{K}) \stackrel{d}{\longrightarrow} \cdots
$$

(b) Its kernel coincides exactly with the submonoid of $\operatorname{Symm}^{(i)}(\mathcal{K})$ made of symmetric $i$-spaces:

$$
\operatorname{MW}^{i}(\mathcal{K})=\operatorname{ker}(d):=d^{-1}(0) .
$$

(c) Its image coincides exactly with the submonoid of $\operatorname{Symm}^{(i+1)}(\mathcal{K})$ made of metabolic or neutral $(i+1)$-spaces:

$$
\mathrm{NW}^{i+1}(\mathcal{K})=\operatorname{im}(d):=d\left(\operatorname{Symm}^{(i)}(\mathcal{K})\right) .
$$

(d) Its homology is the $i$ th triangular Witt group of $\mathcal{K}$ :

$$
\mathrm{W}^{i}(\mathcal{K})=\mathrm{MW}^{i}(\mathcal{K}) / \mathrm{NW}^{i}(\mathcal{K})=\operatorname{ker}(d) / \operatorname{im}(d) .
$$

Proof. The fact that the isometry class of $\operatorname{Cone}(A, a)$ only depends on the isometry class of $(A, a)$ is immediate from the definition, see Definition 2.3 , and the fact that the symmetric space $(C, \Phi)$ constructed there is unique up to isometry [Bal00, Theorem 2.6]. 


\section{P. BALMER}

Part (a) is clear since $\operatorname{Cone}(A, a)$ is a space, so it has a trivial symmetric cone. (In the above notation, we have cone $(\Phi)=0$.) Conversely, $\operatorname{Cone}(A, a)=0$ implies that the form $a$ is an isomorphism, which proves part (b). Parts (c) and (d) are transcriptions of the definitions, see [Bal00, § 2].

Remark 2.6. Observe that the 'homology' of a complex of monoids is probably as slippery a notion as that of 'exact sequence' of monoids. We do not know if there is a reasonable version of the above complex $\operatorname{Symm}^{(\bullet)}(\mathcal{K})$ made of abelian groups (its group completion, for instance) whose homology coincides with the above Witt groups. Although elements in $\operatorname{Symm}^{(i)}(\mathcal{K})$ do not admit an opposite, we can define $-\alpha:=(A,-a)$ for any symmetric pair $\alpha=(A, a)$ in $\operatorname{Symm}^{(i)}(\mathcal{K})$ and we have $d(-\alpha)=-d(\alpha)$. This does provide the opposite in the Witt group.

Remark 2.7. We have 4-periodicity of Witt groups, which is already visible on the level of $\operatorname{Symm}^{(i)}(\mathcal{K})$ and is simply induced by the translation

$$
\begin{aligned}
\operatorname{Symm}^{(i)}(\mathcal{K}) & \stackrel{\sim}{\longrightarrow} \operatorname{Symm}^{(i+4)}(\mathcal{K}) \\
(A, a) & \longmapsto\left(T^{2}(A), T^{2}(a)\right)
\end{aligned}
$$

using that $T D=D T^{-1}$ which gives, in particular, $T^{2} \circ D=D^{(4)} \circ T^{2}$. Note also that the signs involved in $\delta^{(i)}$ and $\varpi^{(i)}$ are 4-periodic, as recalled in Definition A.3.

Example 2.8. Returning to our geometric Examples 1.3 and 1.7, we can now define Witt groups of the respective derived categories with duality associated to the scheme $X$. In case (a), that is for $\mathbb{D}^{\mathrm{b}}\left(\mathrm{VB}_{X}\right)$, and for any line bundle $\mathcal{L} \in \operatorname{Pic}(X)$, we obtain the so-called (derived) Witt groups $\mathrm{W}^{i}(X, \mathcal{L}):=\mathrm{W}^{i}\left(\mathbb{D}^{\mathrm{b}}\left(\mathrm{VB}_{X}\right), D_{\mathcal{L}}\right)$, with $i$-shifted and $\mathcal{L}$-twisted duality, where $D_{\mathcal{L}}$ is the derived duality twisted by $\mathcal{L}$ :

$$
D_{\mathcal{L}}(-)=\mathcal{H o m}_{\mathcal{O}_{X}}\left(-, \mathcal{O}_{X}\right) \otimes \mathcal{L}
$$

The same duality applies in case (c), that is on $\mathbb{D}^{\text {perf }}(X)$, yielding what could be called perfect (derived) Witt groups of $X$. In case (b), that is for $\mathbb{D}^{\mathrm{b}}\left(\mathrm{Coh}_{X}\right)$, and under the assumptions insuring the existence of the duality, we obtain the coherent (derived) Witt groups of $X$, see [Gil02, § 2.5]. We shall not use here the latter two examples but only derived Witt groups (of vector bundles).

\section{Support and degeneracy locus}

Let $\mathcal{K}$ be a triangulated category with duality defined over a topological space $X$ (Definition 1.4). We have the following concepts.

Definition 3.1. We define the support of a symmetric pair $\alpha=(A, a)$ as the support of the object $A$

$$
\operatorname{Supp}(\alpha):=\operatorname{supp}(A) \subset X .
$$

Definition 3.2. We define the degeneracy locus of a symmetric pair $\alpha=(A, a)$ to be the support of the cone of the morphism $a$ :

$$
\operatorname{DegLoc}(\alpha):=\operatorname{supp}(\operatorname{cone}(a)) .
$$

Combined with Proposition 2.5 and Definition 3.1, this gives

$$
\operatorname{DegLoc}(\alpha)=\operatorname{Supp}(d(\alpha)) \text {. }
$$

Proposition 3.3. The degeneracy locus of a symmetric form is always contained in its support: $\operatorname{DegLoc}(\alpha) \subset \operatorname{Supp}(\alpha)$.

Proof. Write $\alpha=(A, a)$ and use the distinguished triangle (3) of Definition 2.3. Then

$$
\operatorname{DegLoc}(\alpha) \stackrel{\text { def }}{=} \operatorname{supp}(C) \stackrel{(\mathrm{S} 4)}{\complement} \operatorname{supp}(A) \cup \operatorname{supp}(D A) \stackrel{(\text { S5 })}{=} \operatorname{supp}(A) \stackrel{\text { def }}{=} \operatorname{Supp}(\alpha) .
$$




\section{Products of DEgENERATE QUADRATIC FORMS}

Proposition 3.4. A symmetric pair is a symmetric space if and only if its degeneracy locus is empty.

Proof. In a triangulated category, a morphism is an isomorphism if and only if its cone is zero. The statement follows from (S1) of Definition 1.1.

Example 3.5. Let $X$ be a scheme and let $s \in \Gamma\left(X, \mathcal{O}_{X}\right)$ be a global section of the structure sheaf $\mathcal{O}_{X}$. Let $\alpha(s)=\left(\mathcal{O}_{X}, s\right)$ be the obvious symmetric pair for the unshifted untwisted duality on $\mathbb{D}^{\mathrm{b}}\left(\mathrm{VB}_{X}\right)$, i.e. consider $\mathcal{O}_{X}$ as a complex concentrated in degree 0 and $s$ as a morphism from $\mathcal{O}_{X}$ to its dual, which is $\mathcal{O}_{X}$ again. Then the support of $\alpha$ is the support of $\mathcal{O}_{X}$, that is, the whole of $X$. The degeneracy locus of $\alpha$ is the zero set $Z(s)$ of $s$. The symmetric cone (Definition 2.3) of $\alpha(s)$ is the following symmetric 1-space $(C, \Phi)$ :

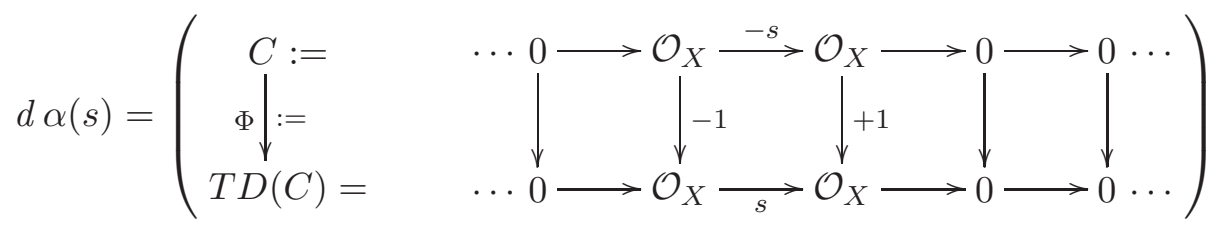

with the objects $\mathcal{O}_{X}$ in homological degrees 1 and 0 . This metabolic space has support $Z(s)$, compare with (5), and its degeneracy locus is empty, as for any space. We shall generalize this example in $\S 6$; see Definition 6.4 and Proposition 6.5.

\section{Product and consanguinity}

In this section, $\square: \mathcal{K} \times \mathcal{L} \rightarrow \mathcal{M}$ is a pairing of triangulated categories with duality defined over $X$ as in Definition 1.6. We write supp for the three support-assignments, independently of the category $\mathcal{K}, \mathcal{L}$ or $\mathcal{M}$

Let $\alpha=(A, a)$ and $\beta=(B, b)$ be two symmetric pairs in $\mathcal{K}$ and $\mathcal{L}$, respectively.

Definition 4.1. We define the product of the symmetric pairs $\alpha$ and $\beta$ to be the symmetric pair $\alpha \star \beta:=\left(A \otimes B, \mu_{A, B} \circ(a \bigotimes b)\right)$. The same notation $\star$ also applies to the form itself. So, we have

$$
(A, a) \star(B, b)=(A \otimes B, a \star b)
$$

where we use $\mu$ to identify the product of the duals with the dual of the product:

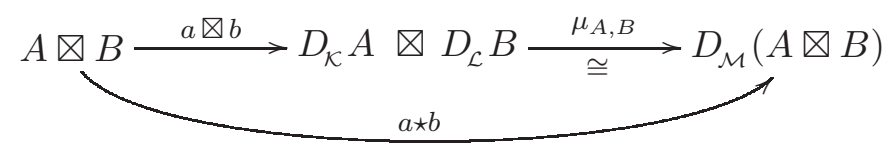

Remark 4.2. See Definition A.6 for how to define $\mu^{(i, j)}$ so that $\left(\bigotimes, \mu^{(i, j)}\right)$ is again a pairing of triangulated categories with duality, when using the $i$-shifted duality $\left(\mathcal{K}, D^{(i)}, \delta^{(i)}, \varpi^{(i)}\right)$ on $\mathcal{K}$, the $j$-shifted duality $\left(\mathcal{L}, D^{(j)}, \delta^{(j)}, \varpi^{(j)}\right)$ on $\mathcal{L}$ and $(i+j)$-shifted duality $\left(\mathcal{M}, D^{(i+j)}, \delta^{(i+j)}, \varpi^{(i+j)}\right)$ on $\mathcal{M}$. With this in mind, Definition 4.1 also applies to the shifted dualities. So, for all $i, j \in \mathbb{Z}$, we have a bi-additive pairing of monoids:

$$
\star: \operatorname{Symm}^{(i)}(\mathcal{K}) \times \operatorname{Symm}^{(j)}(\mathcal{L}) \rightarrow \operatorname{Symm}^{(i+j)}(\mathcal{M}) .
$$

(Distributivity with respect to orthogonal sum, is obvious.) It is clear that this pairing respects 4-periodicity, see Remark 2.7. We shall see in Theorem 5.2 how this pairing behaves with respect to the differential $d: \operatorname{Symm}^{(i)} \rightarrow \operatorname{Symm}^{(i+1)}$.

Definition 4.3. We define the consanguinity of the symmetric pairs $\alpha$ and $\beta$ to be the following closed subset of $X$ :

$$
\operatorname{Cons}(\alpha, \beta):=(\operatorname{Supp}(\alpha) \cap \operatorname{DegLoc}(\beta)) \cup(\operatorname{DegLoc}(\alpha) \cap \operatorname{Supp}(\beta)) .
$$




\section{P. BALMER}

With $\alpha=(A, a), \beta=(B, b)$ and Definitions 3.1 and 3.2, the above subset of $X$ is

$$
\operatorname{Cons}(\alpha, \beta)=(\operatorname{supp}(A) \cap \operatorname{supp}(\operatorname{cone}(b))) \cup(\operatorname{supp}(\operatorname{cone}(a)) \cap \operatorname{supp}(B)) .
$$

We say that the symmetric pairs $\alpha$ and $\beta$ have no consanguinity if $\operatorname{Cons}(\alpha, \beta)=\varnothing$.

Proposition 4.4. We have $\operatorname{Deg} \operatorname{Loc}(\alpha \star \beta) \subset \operatorname{Cons}(\alpha, \beta)$.

Proof. This is the last statement of Lemma 1.10 .

Remark 4.5. In algebraic geometry, we have, in fact, the equality $\operatorname{Deg} \operatorname{Loc}(\alpha \star \beta)=\operatorname{Cons}(\alpha, \beta)$. This can be seen locally via minimal resolutions and we leave it to the interested reader. We do not know whether this can be proved for all triangulated categories defined over a topological space without further assumptions. Anyway, we only need the inclusion of Proposition 4.4 to apply Proposition 3.4 and obtain the following.

Corollary 4.6. If the symmetric pairs $\alpha$ and $\beta$ have no consanguinity then $\alpha \star \beta$ is a symmetric space.

Remark 4.7. We now want to extend the above considerations to products of several symmetric pairs $\alpha_{1} \star \cdots \star \alpha_{n}$. We find it too cumbersome to consider a multiple-entry product $\nabla: \mathcal{K}_{1} \times \cdots \times \mathcal{K}_{n} \longrightarrow \mathcal{M}$ and to unfold all the relevant natural isomorphisms. Therefore, we now restrict attention to TPDcategories $(\mathcal{K}, \otimes)$ defined over $X$ in the sense of Definition 1.6, that is, to the case where all the categories involved coincide. We do not assume the tensor product to be associative, although it will be so in the geometric examples, for the reason that the sign conventions hidden in associativity isomorphisms would overburden the presentation.

Definition 4.8. We extend the definition of the product $\alpha \star \beta$ given in Definition 4.1 to several symmetric pairs by induction over $n \geqslant 2$ :

$$
\alpha_{1} \star \cdots \star \alpha_{n}:=\left(\alpha_{1} \star \cdots \star \alpha_{n-1}\right) \star \alpha_{n} .
$$

Definition 4.9. Let $\alpha_{1}, \ldots, \alpha_{n}$ be symmetric forms in our TPD-category $\mathcal{K}$ defined over the topological space $X$. We define the consanguinity of $\alpha_{1}, \ldots, \alpha_{n}$ to be the following closed subset of $X$ :

$$
\operatorname{Cons}\left(\alpha_{1}, \ldots, \alpha_{n}\right):=\bigcap_{i=1}^{n} \operatorname{Supp}\left(\alpha_{i}\right) \cap\left(\bigcup_{j=1}^{n} \operatorname{DegLoc}\left(\alpha_{j}\right)\right) .
$$

Observe that this definition is symmetric in $\alpha_{1}, \ldots, \alpha_{n}$. We say that the forms $\alpha_{1}, \ldots, \alpha_{n}$ have no consanguinity if this set is empty: $\operatorname{Cons}\left(\alpha_{1}, \ldots, \alpha_{n}\right)=\varnothing$.

LEMma 4.10. Let $\alpha_{1}, \ldots, \alpha_{n}$ be symmetric pairs.

(a) For $n=1$, we have $\operatorname{Cons}\left(\alpha_{1}\right)=\operatorname{DegLoc}\left(\alpha_{1}\right)$.

(b) For $n \geqslant 2$, we have the following inductive formula:

$$
\operatorname{Cons}\left(\alpha_{1}, \ldots, \alpha_{n}\right)=\left(\operatorname{Supp}\left(\alpha_{1} \star \cdots \star \alpha_{n-1}\right) \cap \operatorname{Cons}\left(\alpha_{n}\right)\right) \cup\left(\operatorname{Cons}\left(\alpha_{1}, \ldots, \alpha_{n-1}\right) \cap \operatorname{Supp}\left(\alpha_{n}\right)\right)
$$

which, by statement (a), coincides with Definition 4.3 when $n=2$.

Proof. Statement (a) is immediate from the definition and from Proposition 3.3. Let us compute directly the right-hand side of statement (b):

$$
\begin{aligned}
\operatorname{Supp}\left(\alpha_{1} \star \cdots \star \alpha_{n-1}\right) \cap \operatorname{Cons}\left(\alpha_{n}\right) \cup \operatorname{Cons}\left(\alpha_{1}, \ldots, \alpha_{n-1}\right) \cap \operatorname{Supp}\left(\alpha_{n}\right) \\
\stackrel{(\mathrm{S} 6)}{=} \bigcap_{j=1}^{n-1} \operatorname{Supp}\left(\alpha_{j}\right) \cap \operatorname{Cons}\left(\alpha_{n}\right) \cup \operatorname{Cons}\left(\alpha_{1}, \ldots, \alpha_{n-1}\right) \cap \operatorname{Supp}\left(\alpha_{n}\right) \\
\stackrel{(a)}{\stackrel{\&}{=}}{ }^{4.9} \bigcap_{j=1}^{n-1} \operatorname{Supp}\left(\alpha_{j}\right) \cap \operatorname{DegLoc}\left(\alpha_{n}\right) \cup \bigcap_{j=1}^{n} \operatorname{Supp}\left(\alpha_{j}\right) \cap\left(\bigcup_{i=1}^{n-1} \operatorname{DegLoc}\left(\alpha_{i}\right)\right)
\end{aligned}
$$


Products of Degenerate QuAdratic Forms

$$
\begin{aligned}
& \stackrel{3.3}{=} \bigcap_{j=1}^{n} \operatorname{Supp}\left(\alpha_{j}\right) \cap \operatorname{DegLoc}\left(\alpha_{n}\right) \cup \bigcap_{j=1}^{n} \operatorname{Supp}\left(\alpha_{j}\right) \cap\left(\bigcup_{i=1}^{n-1} \operatorname{DegLoc}\left(\alpha_{i}\right)\right) \\
& \stackrel{4.9}{=} \operatorname{Cons}\left(\alpha_{1}, \ldots, \alpha_{n}\right) .
\end{aligned}
$$

Proposition 4.11. Let $\alpha_{1}, \ldots, \alpha_{n}$ be symmetric forms. Then, we have

$$
\operatorname{DegLoc}\left(\alpha_{1} \star \cdots \star \alpha_{n}\right) \subset \operatorname{Cons}\left(\alpha_{1}, \ldots, \alpha_{n}\right) .
$$

Proof. By induction over $n$. For $n=1$ both sides are equal to $\operatorname{DegLoc}\left(\alpha_{1}\right)$ by Lemma $4.10(a)$. Assume that $n \geqslant 2$ and that the result holds for $n-1$. We have

$$
\begin{aligned}
& \operatorname{DegLoc}\left(\alpha_{1} \star \cdots \star \alpha_{n}\right) \stackrel{4.4}{\subset} \operatorname{Cons}\left(\left(\alpha_{1} \star \cdots \star \alpha_{n-1}\right), \alpha_{n}\right) \\
& \stackrel{4.3}{=} \operatorname{Supp}\left(\alpha_{1} \star \cdots \star \alpha_{n-1}\right) \cap \operatorname{DegLoc}\left(\alpha_{n}\right) \cup \operatorname{DegLoc}\left(\alpha_{1} \star \cdots \star \alpha_{n-1}\right) \cap \operatorname{Supp}\left(\alpha_{n}\right) \\
& \stackrel{\text { I.H. }}{\subset} \operatorname{Supp}\left(\alpha_{1} \star \cdots \star \alpha_{n-1}\right) \cap \operatorname{DegLoc}\left(\alpha_{n}\right) \cup \operatorname{Cons}\left(\alpha_{1}, \ldots, \alpha_{n-1}\right) \cap \operatorname{Supp}\left(\alpha_{n}\right) \\
& \stackrel{4.10}{=} \operatorname{Cons}\left(\alpha_{1}, \ldots, \alpha_{n}\right),
\end{aligned}
$$

where the inclusion labelled 'I.H.' holds by the induction hypothesis.

Remark 4.12. The above proof shows that if equality holds in Proposition 4.4 then it holds in Proposition 4.11 as well. This is, in particular, the case in algebraic geometry as mentioned in Remark 4.5. Still, we only need the above inclusion for the following.

Corollary 4.13. Let $\alpha_{1}, \ldots, \alpha_{n}$ be symmetric forms with no consanguinity. Then $\alpha_{1} \star \cdots \star \alpha_{n}$ is non-degenerate.

\section{Leibniz formula}

We return to the general situation of a pairing $\nabla: \mathcal{K} \times \mathcal{L} \longrightarrow \mathcal{M}$ of triangulated categories with duality, defined over a topological space $X$, as in Definition 1.6.

Proposition 5.1. Let $\alpha$ and $\beta$ be symmetric forms. Then the following are equivalent.

(a) The degeneracy loci of the forms $\alpha$ and $\beta$ do not intersect.

(b) The forms $d(\alpha)$ and $\beta$ have no consanguinity.

(c) The forms $\alpha$ and $d(\beta)$ have no consanguinity.

In this case, we say that $\alpha$ and $\beta$ have no common degeneracy, which implies, in particular, that $d(\alpha) \star \beta$ and $\alpha \star d(\beta)$ are non-degenerate.

Proof. We have $\operatorname{DegLoc}(d(\alpha))=\varnothing$ and $\operatorname{Supp}(d(\alpha))=\operatorname{DegLoc}(\alpha)$, see (5). By Definition 4.3, it follows that $\operatorname{Cons}(d(\alpha), \beta)=\operatorname{DegLoc}(\alpha) \cap \operatorname{DegLoc}(\beta)=\operatorname{Cons}(\alpha, d(\beta))$. This proves the equivalence of the three conditions. The conclusion about the non-degeneracy of the products comes from Corollary 4.6.

For the next result, we need to assume that the pairing $\nabla: \mathcal{K} \times \mathcal{L} \longrightarrow \mathcal{M}$ is compatible with the octahedron axiom, as recalled in Definition A.11. This axiomatization follows May [May01] and holds of course for any pairing observable in nature.

Theorem 5.2 (Leibniz-type formula for symmetric spaces). Let $\alpha$ and $\beta$ be symmetric forms with no common degeneracy (see Proposition 5.1). Then, we have an isometry

$$
\delta_{\mathcal{M}} \cdot d(\alpha \star \beta) \simeq \delta_{\mathcal{K}} \cdot d(\alpha) \star \beta+\delta_{\mathcal{L}} \cdot \alpha \star d(\beta),
$$

where we recall that the signs $\delta_{\mathcal{K}}, \delta_{\mathcal{L}}, \delta_{\mathcal{M}}= \pm 1$ express the exactness of the three dualities involved $D_{\mathcal{K}}, D_{\mathcal{L}}$ and $D_{\mathcal{M}}$. 


\section{P. BALMER}

Proof. Write the symmetric forms $\alpha=(A, a)$ and $\beta=(B, b)$ and consider distinguished triangles in $\mathcal{K}$ and $\mathcal{L}$, respectively,

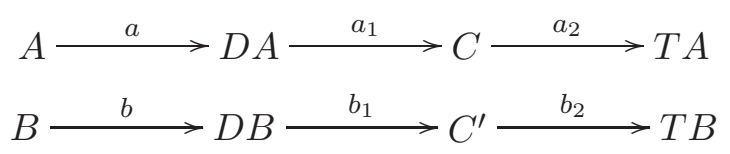

as well as the cone symmetric forms $\Phi: C \stackrel{\sim}{\rightarrow} D_{\mathcal{K}}^{(1)}(C)$ and $\Phi^{\prime}: C^{\prime} \stackrel{\sim}{\rightarrow} D_{\mathcal{L}}^{(1)}\left(C^{\prime}\right)$ which satisfy the following equations (see (4) in Definition 2.3 if necessary):

$$
\Phi \circ a_{1}=-D_{\mathcal{K}}^{(1)}\left(a_{2}\right) \text { and } \Phi^{\prime} \circ b_{1}=-D_{\mathcal{L}}^{(1)}\left(b_{2}\right) .
$$

From now on, we shall write $D$ for $D_{\mathcal{K}}, D_{\mathcal{L}}$ and $D_{\mathcal{M}}$ since it is always clear which duality is meant from the object or the morphism it is applied to.

The proof will consist of finding a distinguished triangle over the morphism $a \star b$ and of showing that the symmetric form $\Phi^{\prime \prime}$ on its cone satisfying an equation of type (8) can be chosen to be $\Phi^{\prime \prime}=\delta_{\mathcal{K}} \delta_{\mathcal{M}} \cdot(\Phi \star b) \perp \delta_{\mathcal{L}} \delta_{\mathcal{M}} \cdot\left(a \star \Phi^{\prime}\right)$ as announced in the statement. This will be the symmetric cone $d(\alpha \star \beta)$ by uniqueness of the construction, see Definition 2.3. Indeed, it would not be hard to prove directly that the cone of $a \star b$, which is the cone of $a \otimes b$, is isomorphic to the direct sum of $C \otimes B$ and $A \otimes C^{\prime}$ as predicted by the Theorem. What is harder is to get the right morphisms in this distinguished triangle in order to check the equation of type (4) for $\Phi^{\prime \prime}$ and such a triangle is what we are now going to establish.

The assumption $\varnothing=\operatorname{DegLoc}(\alpha) \cap \operatorname{DegLoc}(\beta)=\operatorname{supp}(C) \cap \operatorname{supp}\left(C^{\prime}\right) \stackrel{(\mathrm{S} 6)}{=} \operatorname{supp}\left(C \otimes C^{\prime}\right) \operatorname{implies}$ by (S1) the vanishing of the product $C \otimes C^{\prime}=0$. We use this in the next diagram. Applying the bi-exact functor $-\nabla-$ to the above distinguished triangles (6) and (7), we obtain a diagram with distinguished rows and columns, which commutes except for the lower-right square which anti-commutes:

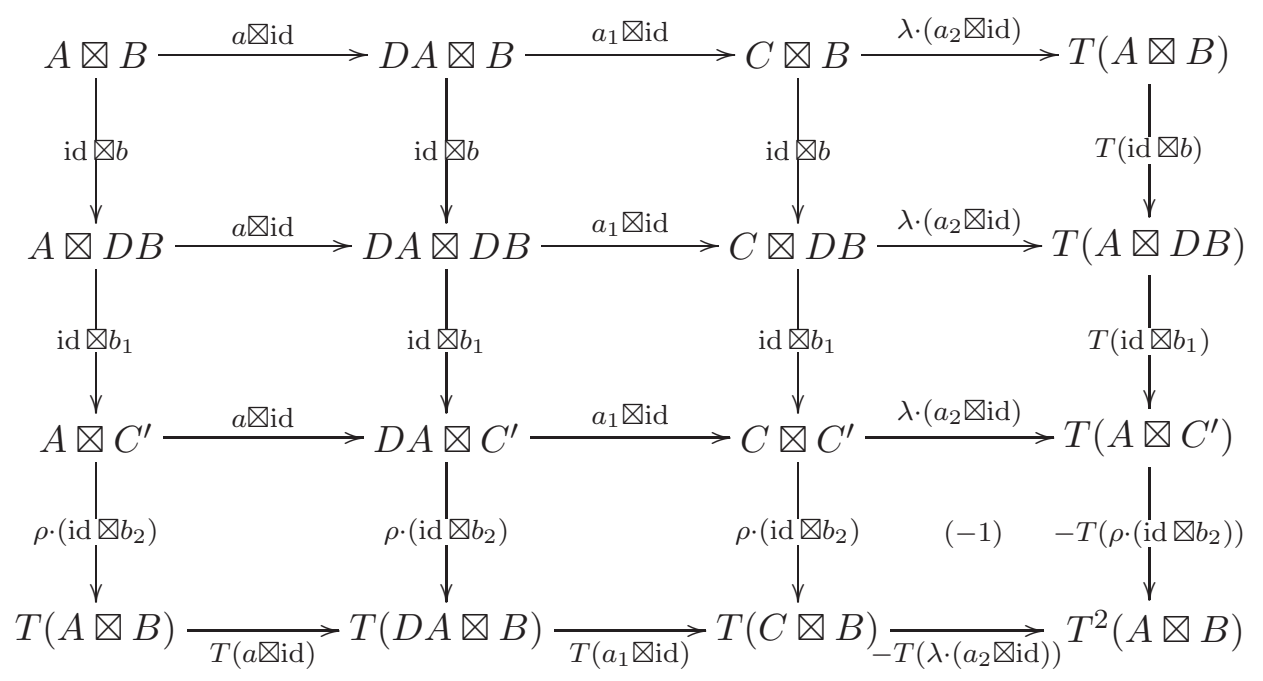

Plugging $C \otimes C^{\prime}=0$ in this diagram, we immediately deduce the following:

$$
\begin{gathered}
\bar{a}:=a \otimes \operatorname{id}_{C^{\prime}}: A \otimes C^{\prime} \stackrel{\sim}{\longrightarrow} D A \otimes C^{\prime} \quad \text { is an isomorphism } \\
\bar{b}:=\operatorname{id}_{C} \otimes b: C \otimes B \stackrel{\sim}{\longrightarrow} C \otimes D B \quad \text { is an isomorphism }
\end{gathered}
$$

from exactness of the third rows and columns, whereas commutativity of the squares ' $(2,3)$ ' and ' $(3,2)$ ' gives

$$
a_{2} \otimes b_{1}=0 \quad \text { and } \quad a_{1} \otimes b_{2}=0 .
$$




\section{Products of DEgenerate QUADRATIC FORMS}

From compatibility of the pairing $\nabla$ with the octahedron axiom (Definition A.11) there exist in $\mathcal{M}$ three distinguished octahedra:
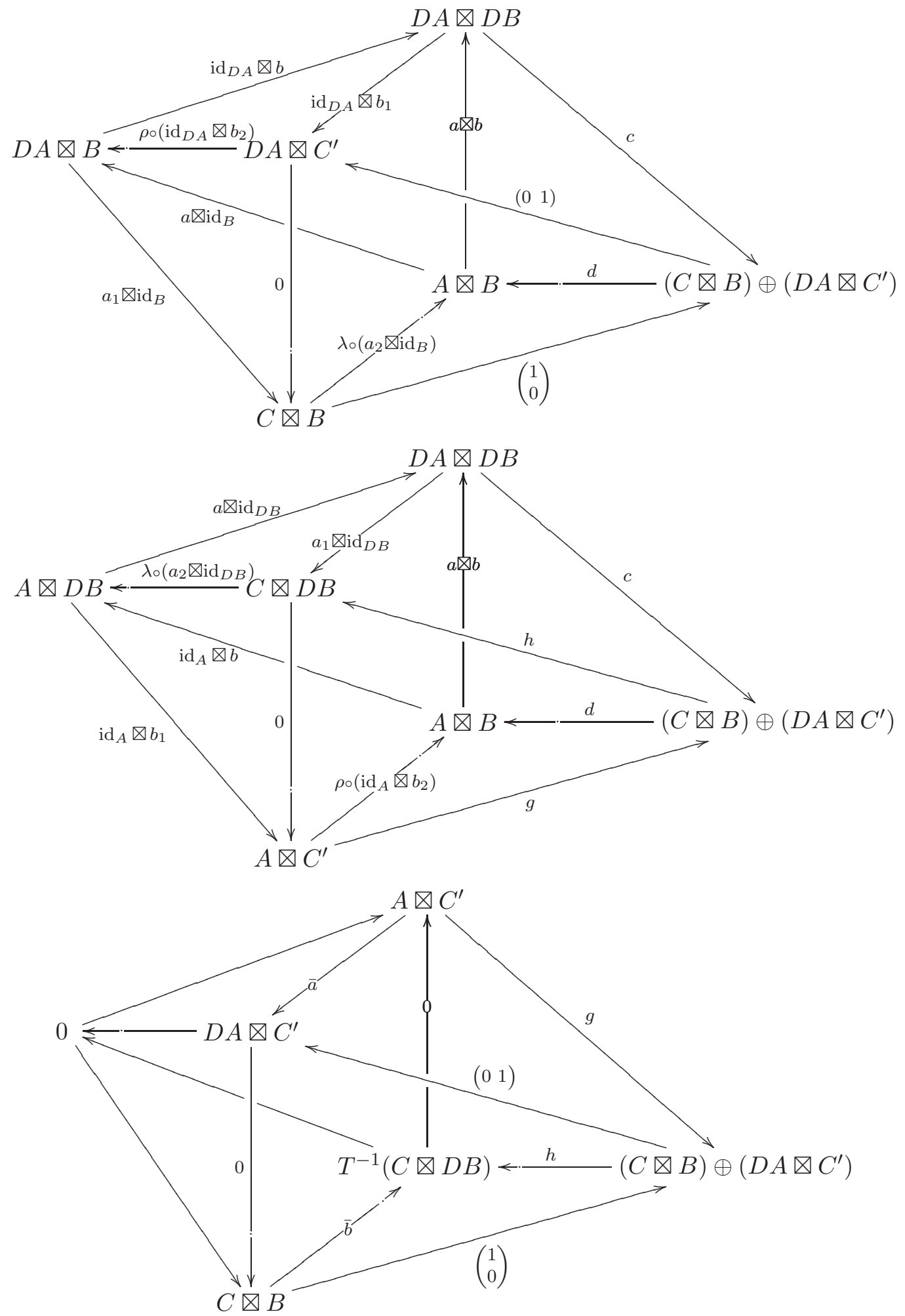

These three octahedra are simply (37), (38) and (39) of Definition A.11 applied to the distinguished triangles (6) and (7), in which we used the above information $C \otimes C^{\prime}=0$, as well as $a_{2} \otimes b_{1}=0$ and $a_{1} \otimes b_{2}=0$. As explained in Remark A.13, we also allowed ourselves to choose one 


\section{P. BALMER}

of the distinguished triangles (34), (35), (36), namely (35), that over $a_{1} \otimes b_{2}=0$, which we chose to be

$$
C \otimes B \stackrel{e:=\left(\begin{array}{l}
1 \\
0
\end{array}\right)}{\longrightarrow}(C \otimes B) \oplus\left(D A \otimes C^{\prime}\right) \stackrel{f:=\left(\begin{array}{ll}
0 & 1
\end{array}\right)}{\longrightarrow} D A \otimes C^{\prime} \stackrel{0}{\longrightarrow} T(C \otimes B) .
$$

This is also how we know that the object $E$ of Definition A.11 is here $(C \otimes B) \oplus\left(D A \otimes C^{\prime}\right)$.

From the commutativity in the third octahedron, we immediately compute one entry, in matrix notation, of each of the morphisms $g$ and $h$. Since $h \circ g=0$ and since $\bar{a}$ and $\bar{b}$ are isomorphisms, we deduce that there exists a morphism

$$
k: A \otimes C^{\prime} \rightarrow C \otimes B \quad \text { such that } g=\left(\begin{array}{l}
k \\
\bar{a}
\end{array}\right) \text { and } h=\left(\begin{array}{ll}
\bar{b} & -\bar{b} k \bar{a}^{-1}
\end{array}\right) .
$$

We are now going to use the trick of Remark A.13 again, namely that explained in the second part of Remark A.13, carefully keeping our two morphisms $e=\left(\begin{array}{l}1 \\ 0\end{array}\right)$ and $f=\left(\begin{array}{ll}0 & 1\end{array}\right)$. For this, consider the automorphism

$$
\ell:=\left(\begin{array}{cc}
1 & -k \bar{a}^{-1} \\
0 & 1
\end{array}\right):(C \otimes B) \oplus\left(D A \otimes C^{\prime}\right) \stackrel{\sim}{\longrightarrow}(C \otimes B) \oplus\left(D A \otimes C^{\prime}\right) .
$$

Composing the above octahedra with this isomorphism $\ell$ gives three new octahedra, which are of course as distinguished as the above octahedra. Let us see what happens to the morphisms involved in this composition, namely the six morphisms having source or target equal to the modified object. They become

$$
\ell \circ\left(\begin{array}{l}
1 \\
0
\end{array}\right), \quad\left(\begin{array}{ll}
0 & 1
\end{array}\right) \circ \ell^{-1}, \quad \ell \circ c, \quad d \circ \ell^{-1}, \quad \ell \circ g \quad \text { and } h \circ \ell^{-1}
$$

Now, by choice of the automorphism $\ell$ and by (13) these six morphisms simply are

$$
\left(\begin{array}{l}
1 \\
0
\end{array}\right), \quad\left(\begin{array}{ll}
0 & 1
\end{array}\right), \quad \ell \circ c, \quad d \circ \ell^{-1}, \quad\left(\begin{array}{l}
0 \\
\bar{a}
\end{array}\right) \quad \text { and }\left(\begin{array}{ll}
\bar{b} & 0
\end{array}\right) \text {. }
$$

Let us rebaptise the last four morphisms $c, d, g$ and $h$, respectively. So, we now have three octahedra exactly as above, with, in addition,

$$
g=\left(\begin{array}{l}
0 \\
\bar{a}
\end{array}\right) \quad \text { and } \quad h=\left(\begin{array}{ll}
\bar{b} & 0
\end{array}\right) .
$$

Using this in the second octahedron it follows that

$$
c=\left(\begin{array}{c}
\bar{b}^{-1} \circ\left(a_{1} \otimes \operatorname{id}_{D B}\right) \\
?
\end{array}\right) \text { and } \quad d=\left(? ? \quad \rho \circ\left(\operatorname{id}_{A} \otimes b_{2}\right) \circ \bar{a}^{-1}\right)
$$

whereas the first octahedron gives us

$$
c=\left(\begin{array}{c}
? ? ? \\
\operatorname{id}_{D A} \otimes b_{1}
\end{array}\right) \quad \text { and } \quad d=\left(\lambda \circ\left(a_{2} \otimes \operatorname{id}_{B}\right) \quad ? ? ? ?\right) .
$$

Since it is the same $c$ and the same $d$ in both octahedra (this is the whole point of this proof!), we can put (14) and (15) together and obtain

$$
c=\left(\begin{array}{c}
\bar{b}^{-1} \circ\left(a_{1} \otimes \operatorname{id}_{D B}\right) \\
\operatorname{id}_{D A} \otimes b_{1}
\end{array}\right) \quad \text { and } \quad d=\left(\lambda \circ\left(a_{2} \otimes \operatorname{id}_{B}\right) \quad \rho \circ\left(\operatorname{id}_{A} \otimes b_{2}\right) \circ \bar{a}^{-1}\right),
$$

that is, we have the complete description of a distinguished triangle over $a \bigotimes b$ :

$$
A \otimes B \stackrel{a \bigotimes b}{\longrightarrow} D A \otimes D B \stackrel{c}{\longrightarrow}(C \otimes B) \oplus\left(D A \otimes C^{\prime}\right) \stackrel{d}{\longrightarrow} T(A \otimes B) .
$$




\section{Products of Degenerate Quadratic Forms}

From this we deduce the distinguished triangle over $a \star b=\mu_{A, B} \circ a \otimes b$ in the obvious way, since $\mu_{A, B}: D A \otimes D B \stackrel{\sim}{\rightarrow} D(A \otimes B)$ is an isomorphism

$$
A \otimes B \stackrel{a \star b}{\longrightarrow} D(A \otimes B) \stackrel{m_{1}}{\longrightarrow}(C \otimes B) \oplus\left(A \otimes C^{\prime}\right) \stackrel{m_{2}}{\longrightarrow} T(A \otimes B),
$$

in which we also replaced $D A \otimes C^{\prime}$ by the isomorphic $A \otimes C^{\prime}$, using the isomorphism $\bar{a}$. The morphisms $m_{1}$ and $m_{2}$ are explicitly given by

$$
m_{1}=\left(\begin{array}{cc}
\text { id } & 0 \\
0 & \bar{a}^{-1}
\end{array}\right) \circ c \circ \mu^{-1} \stackrel{(16)}{=}\left(\begin{array}{c}
\bar{b}^{-1} \circ\left(a_{1} \otimes \mathrm{id}_{D B}\right) \\
\bar{a}^{-1} \circ\left(\operatorname{id}_{D A} \nabla b_{1}\right)
\end{array}\right) \circ \mu^{-1},
$$

and

$$
m_{2}=d \circ\left(\begin{array}{cc}
\mathrm{id} & 0 \\
0 & \bar{a}
\end{array}\right) \stackrel{(16)}{=}\left(\lambda \circ\left(a_{2} \otimes \mathrm{id}_{B}\right) \quad \rho \circ\left(\operatorname{id}_{A} \nabla b_{2}\right)\right) .
$$

Consider now the two symmetric 1-forms:

$$
\Phi \star b: C \otimes B \stackrel{\sim}{\longrightarrow} D^{(1)}(C \otimes B) \quad \text { and } \quad a \star \Phi^{\prime}: A \otimes C^{\prime} \stackrel{\sim}{\longrightarrow} D^{(1)}\left(A \otimes C^{\prime}\right)
$$

which are non-degenerate by Proposition 5.1. The claim of the Theorem is that their orthogonal sum $\Phi^{\prime \prime}$ (up to the signs $\delta_{\mathcal{K}}, \delta_{\mathcal{L}}, \delta_{\mathcal{M}}$ announced in the statement)

$$
\begin{gathered}
\Phi^{\prime \prime}:=\delta_{\mathcal{K}} \delta_{\mathcal{M}} \cdot(\Phi \star b) \perp \delta_{\mathcal{L}} \delta_{\mathcal{M}} \cdot\left(a \star \Phi^{\prime}\right) \\
\Phi^{\prime \prime}:(C \otimes B) \oplus\left(A \otimes C^{\prime}\right) \stackrel{\sim}{\longrightarrow} D^{(1)}\left((C \otimes B) \oplus\left(A \otimes C^{\prime}\right)\right)
\end{gathered}
$$

is isomorphic to Cone $(a \star b)$. To check this, using the definition of the symmetric cone in Definition 2.3, we have to find a distinguished triangle over $a \star b$, which we indeed already have in (17), and we then have to establish the analogue of (4), namely

$$
\Phi^{\prime \prime} \circ m_{1}=-D^{(1)}\left(m_{2}\right)
$$

or, equivalently, since $\mu_{A, B}: D A \otimes D B \stackrel{\sim}{\longrightarrow} D(A \otimes B)$ is an isomorphism,

$$
\Phi^{\prime \prime} \circ m_{1} \circ \mu=-D^{(1)}\left(m_{2}\right) \circ \mu \text {. }
$$

To show this, first observe that

$$
\Phi \star b \stackrel{\text { def }}{=} \mu \circ(\Phi \otimes b)=\mu \circ\left(\Phi \otimes \operatorname{id}_{D B}\right) \circ\left(\operatorname{id}_{C} \otimes b\right) \stackrel{(11)}{=} \mu \circ\left(\Phi \otimes \operatorname{id}_{D B}\right) \circ \bar{b} .
$$

Similarly, using (10), we get that

$$
a \star \Phi^{\prime}=\mu \circ\left(\operatorname{id}_{D A} \otimes \Phi^{\prime}\right) \circ \bar{a} .
$$

Hence, the left-hand side of (21) becomes, in matrix notation,

$$
\begin{aligned}
\Phi^{\prime \prime} \circ m_{1} \circ \mu & \stackrel{(20)}{=}\left(\delta_{\mathcal{K}} \delta_{\mathcal{M}} \cdot \Phi \star b \perp \delta_{\mathcal{L}} \delta_{\mathcal{M}} \cdot a \star \Phi^{\prime}\right) \circ m_{1} \circ \mu \\
& \stackrel{(18)}{=}\left(\begin{array}{cc}
\delta_{\mathcal{K}} \delta_{\mathcal{M}} \cdot \Phi \star b & 0 \\
0 & \delta_{\mathcal{L}} \delta_{\mathcal{M}} \cdot a \star \Phi^{\prime}
\end{array}\right) \cdot\left(\begin{array}{l}
\bar{b}^{-1} \circ\left(a_{1} \otimes \mathrm{id}_{D B}\right) \\
\bar{a}^{-1} \circ\left(\operatorname{id}_{D A} \nabla b_{1}\right)
\end{array}\right) \\
& \stackrel{(22),(23)}{=}\left(\begin{array}{l}
\delta_{\mathcal{K}} \delta_{\mathcal{M}} \cdot \mu \circ\left(\Phi \otimes \operatorname{id}_{D B}\right) \circ\left(a_{1} \otimes \operatorname{id}_{D B}\right) \\
\delta_{\mathcal{L}} \delta_{\mathcal{M}} \cdot \mu \circ\left(\operatorname{id}_{D A} \nabla \Phi^{\prime}\right) \circ\left(\operatorname{id}_{D A} \nabla b_{1}\right)
\end{array}\right) \\
& \stackrel{(8)}{=}-\left(\begin{array}{l}
\delta_{\mathcal{K}} \delta_{\mathcal{M}} \cdot \mu \circ\left(D^{(1)}\left(a_{2}\right) \otimes \operatorname{id}_{D B}\right) \\
\delta_{\mathcal{L}} \delta_{\mathcal{M}} \cdot \mu \circ\left(\operatorname{id}_{D A} \otimes D^{(1)}\left(b_{2}\right)\right)
\end{array}\right) .
\end{aligned}
$$

We are almost done except that we need to move the natural isomorphism $\mu$ around. At this stage, it is necessary to add decorations $\mu^{(i, j)}$ to specify the considered natural isomorphism 


\section{P. BALMER}

between $D^{(i)}(-) \otimes D^{(j)}(-)$ and $D^{(i+j)}(-\otimes-)$, induced by $\mu^{(0,0)}=\mu$ as defined in Definition A.6. Otherwise, it is impossible to understand the appearance of the signs. We have indeed established

$$
\Phi^{\prime \prime} \circ m_{1} \circ \mu=-\left(\begin{array}{l}
\delta_{\mathcal{K}} \delta_{\mathcal{M}} \cdot \mu^{(1,0)} \circ\left(D^{(1)}\left(a_{2}\right) \otimes \operatorname{id}_{D B}\right) \\
\delta_{\mathcal{L}} \delta_{\mathcal{M}} \cdot \mu^{(0,1)} \circ\left(\operatorname{id}_{D A} \otimes D^{(1)}\left(b_{2}\right)\right)
\end{array}\right) .
$$

Using naturality of the transformation $\mu^{(i, j)}$ our left-hand side of $(21)$ becomes

$$
\begin{aligned}
\Phi^{\prime \prime} \circ m_{1} \circ \mu & =-\left(\begin{array}{l}
\delta_{\mathcal{K}} \delta_{\mathcal{M}} \cdot D^{(1)}\left(a_{2} \otimes \mathrm{id}_{B}\right) \circ \mu_{T A, B}^{(1,0)} \\
\delta_{\mathcal{L}} \delta_{\mathcal{M}} \cdot D^{(1)}\left(\mathrm{id}_{A} \otimes b_{2}\right) \circ \mu_{A, T B}^{(0,1)}
\end{array}\right) \\
& \stackrel{\text { A. } 6}{=}-\left(\begin{array}{l}
D^{(1)}\left(a_{2} \otimes \mathrm{id}_{B}\right) \circ \delta_{\mathcal{K}} \delta_{\mathcal{M}} \cdot T\left(\mu_{T A, B}\right) \circ \lambda_{D T A, D B} \\
D^{(1)}\left(\mathrm{id}_{A} \otimes b_{2}\right) \circ \delta_{\mathcal{L}} \delta_{\mathcal{M}} \cdot T\left(\mu_{A, T B}\right) \circ \rho_{D A, D T B}
\end{array}\right) \\
& \stackrel{\mathrm{PD} 2) \text { in A.4 }}{=}-\left(\begin{array}{c}
D^{(1)}\left(a_{2} \otimes \mathrm{id}_{B}\right) \circ D^{(1)}\left(\lambda_{A, B}\right) \circ \mu_{A, B} \\
D^{(1)}\left(\mathrm{id}_{A} \otimes b_{2}\right) \circ D^{(1)}\left(\rho_{A, B}\right) \circ \mu_{A, B}
\end{array}\right) \\
& =-\left(\begin{array}{c}
D^{(1)}\left(\lambda_{A, B} \circ\left(a_{2} \otimes \mathrm{id}_{B}\right)\right) \\
D^{(1)}\left(\rho_{A, B} \circ\left(\mathrm{id}_{A} \otimes b_{2}\right)\right)
\end{array}\right) \circ \mu_{A, B} \stackrel{(19)}{=}-D^{(1)}\left(m_{2}\right) \circ \mu_{A, B} .
\end{aligned}
$$

This establishes the wanted equation (21) and finishes the proof.

Corollary 5.3. Suppose that $\alpha$ and $\beta$ have no common degeneracy (Proposition 5.1). Then the two symmetric spaces $d(\alpha) \star \beta$ and $\alpha \star d(\beta)$ define, up to a sign, the same Witt class

$$
[d(\alpha) \star \beta]=-\delta_{\mathcal{K}} \delta_{\mathcal{L}} \cdot[\alpha \star d(\beta)]
$$

in the suitable Witt group of $\mathcal{M}$.

Remark 5.4. Of course, if we choose $\beta$ to be non-degenerate in Theorem 5.2 , the formula simply says

$$
d(\alpha \star \beta)= \pm d(\alpha) \star \beta .
$$

This also proves that $d(\alpha) \star \beta$ is metabolic for any non-degenerate symmetric form $\beta$, i.e. that $\star$ induces a well-defined product $\star$ on Witt groups, as already established in [GN03]. The linearity of the connecting homomorphism in the localization long exact sequences follows from this same equation. The verification of the details is left to the reader, simplifying slightly [GN03, $§ 2.3$.

\section{Pseudo-diagonal forms}

Notation 6.1. In this section, we move towards geometric applications. So, we fix a $\mathbb{Z}\left[\frac{1}{2}\right]$-scheme $X$ and, as explained in Examples 1.3(a), 1.7 and 2.8, we consider the bounded derived category $\mathbb{D}^{\mathrm{b}}\left(\mathrm{VB}_{X}\right)$ of vector bundles over $X$ as a TPD-category (Definition A.5) with the usual product $\otimes_{\mathcal{O}_{X}}$. Here, support, degeneracy locus and consanguinity are closed subsets of the underlying topological space of $X$. We shall consider dualities twisted by various line bundles $\mathcal{L} \in \operatorname{Pic}(X)$. Recall that the whole theory is 2-periodic in the twists, exactly as for classical dualities, and 4-periodic in the shifts, see Remark 2.7. So, if we abbreviate the monoid of symmetric pairs (Definition 2.2) for the triangulated category with $\mathcal{L}$-twisted duality $\left(\mathbb{D}^{\mathrm{b}}\left(\mathrm{VB}_{X}\right), D_{\mathcal{L}}\right)$ by

$$
\operatorname{Symm}^{(i)}(X, \mathcal{L}):=\operatorname{Symm}^{(i)}\left(\mathbb{D}^{\mathrm{b}}\left(\mathrm{VB}_{X}\right), D_{\mathcal{L}}\right),
$$




\section{Products of DEgenerate QUAdratic Forms}

we obtain a bi-graded monoid

$$
\operatorname{Symm}^{\operatorname{Tot}}(X):=\bigoplus_{i \in \mathbb{Z} / 4} \bigoplus_{\mathcal{L} \in \operatorname{Pic}(X) / 2} \operatorname{Symm}^{(i)}(X, \mathcal{L})
$$

and the product of symmetric pairs of Definition 4.1 defines a product

$$
\begin{aligned}
\star: \operatorname{Symm}^{(i)}\left(X, \mathcal{L}_{1}\right) \times \operatorname{Symm}^{(j)}\left(X, \mathcal{L}_{2}\right) & \longrightarrow \operatorname{Symm}^{(i+j)}\left(X, \mathcal{L}_{1} \otimes \mathcal{L}_{2}\right) \\
(\alpha, \beta) & \longmapsto \alpha \star \beta .
\end{aligned}
$$

Recall from Definition 4.8 that the product of several symmetric pairs is $\alpha_{1} \star \cdots \star \alpha_{n}=\left(\alpha_{1} \star \cdots \star\right.$ $\left.\alpha_{n-1}\right) \star \alpha_{n}$, with shifts and twists adding up as above.

Remark 6.2. It might be useful to think of $\operatorname{Symm}^{\text {Tot }}(X)$, equipped with orthogonal sum and product, as a graded semiring, i.e. almost a graded ring but with no opposite for the addition. Note that, although it comes equipped with a differential (the symmetric cone $d$ of Proposition 2.5), $\operatorname{Symm}^{\mathrm{Tot}}(X)$ is not a differential graded semiring since the Leibniz rule only holds conditionally, by Theorem 5.2 .

We do not formalize commutativity and associativity of $\otimes$ in the abstract triangular framework, but they hold in this geometric situation for $\otimes_{\mathcal{O}_{X}}$. Note, however, that signs might be involved in the associativity of the product, depending on conventions. We renounce these rather arid considerations here, since we can moreover circumvent them in applications, see Remark 7.8. So, we leave the proof of the following rather obvious statement to the careful reader. For $\langle 1\rangle$, recall that it is the symmetric pair $\left(\mathcal{O}_{X}, 1\right)$ which is clearly a unit for $\star$.

Lemma 6.3. Up to signs (in the sense of Remark 2.6), the product on $\operatorname{Symm}^{\mathrm{Tot}}(X)$ is commutative and associative. Moreover, it admits a unit $\langle 1\rangle \in \operatorname{Symm}^{(0)}\left(X, \mathcal{O}_{X}\right)$.

We generalize Example 3.5 as follows.

Definition 6.4. Let $\mathcal{L}$ be a line bundle and let $s: \mathcal{O}_{X} \rightarrow \mathcal{L}$ be a global section.

(a) We denote by $Z(s) \subset X$ the zero locus of $s$, that is, the smallest closed subset of $X$ outside of which $s$ is an isomorphism:

$$
\left.s\right|_{X \backslash Z(s)}:\left.\mathcal{O}_{X \backslash Z(s)} \stackrel{\sim}{\longrightarrow} \mathcal{L}\right|_{X \backslash Z(s)} .
$$

(b) We denote by $\alpha(s ; \mathcal{L}):=\left(\mathcal{O}_{X}, s\right) \in \operatorname{Symm}^{(0)}(X, \mathcal{L})$ the symmetric pair

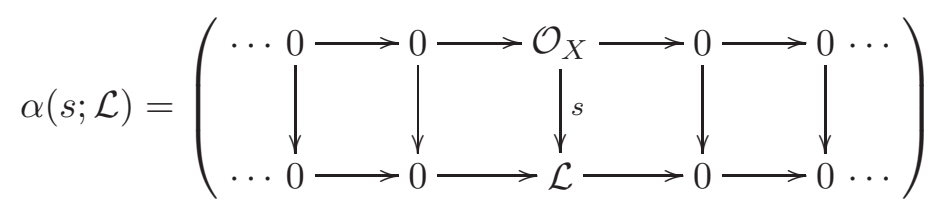

formed by the object $\mathcal{O}_{X}$, considered in $\mathbb{D}^{\mathrm{b}}\left(\mathrm{VB}_{X}\right)$ as a complex concentrated in degree 0 , and by the form $s: \mathcal{O}_{X} \rightarrow D_{\mathcal{L}}\left(\mathcal{O}_{X}\right)=\mathcal{L}$. For simplicity, we might write $\alpha(s)$ instead of $\alpha(s ; \mathcal{L})$.

(c) We call diagonal symmetric pair any (orthogonal) sum of symmetric pairs as above $\alpha\left(s_{1} ; \mathcal{L}_{1}\right)+\cdots+\alpha\left(s_{n} ; \mathcal{L}_{n}\right) \in \operatorname{Symm}^{\operatorname{Tot}}(X)$.

Proposition 6.5. With the above notation, the symmetric cone of $\alpha(s ; \mathcal{L})$ is

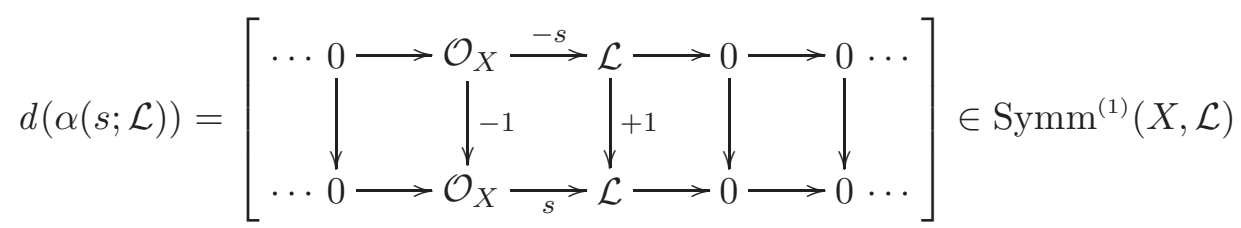




\section{P. BALMER}

with $\mathcal{L}$ in degree 0 . (The object is the complex in the first row, its $D_{\mathcal{L}}^{(1)}$-dual the object in the second row and the symmetric 1-form the vertical morphism of complexes.) Therefore,

$$
\begin{gathered}
\operatorname{Supp}(\alpha(s))=X \quad \operatorname{DegLoc}(\alpha(s))=Z(s) \\
\operatorname{Supp}(d \alpha(s))=Z(s) \quad \operatorname{DegLoc}(d \alpha(s))=\varnothing .
\end{gathered}
$$

Proof. By definition, the cone $C$ of the morphism $s$ is the complex depicted in the first row, see [Wei94, $\S 1.5]$. One has to check that the vertical morphism defines the form $\Phi: C \rightarrow T D_{\mathcal{L}}(C)$ of formula (4) in Definition 2.3. This is easy since the morphism $a_{1}$ is $\mathrm{id}_{\mathcal{L}}$ in degree zero and 0 elsewhere, whereas $a_{2}$ is $-\operatorname{id}_{\mathcal{O}_{X}}$ in degree 1 and zero elsewhere, with the same convention [Wei94, 1.5.2].

Therefore, $\operatorname{Supp}(\alpha(s ; \mathcal{L})) \stackrel{3.1}{=} \operatorname{supp}\left(\mathcal{O}_{X}\right)=X$. The next two equalities of the statement come from $\operatorname{DegLoc}(\alpha(s ; \mathcal{L})) \stackrel{(5)}{=} \operatorname{Supp}(d \alpha(s ; \mathcal{L})) \stackrel{3.1}{=} \operatorname{supp}(\operatorname{cone}(s)) \stackrel{6.4(a)}{=} Z(s)$. Finally, $d(\alpha)$ is non-degenerate for any symmetric pair $\alpha$.

Definition 6.6. We denote by

$$
\operatorname{PDiag}^{\operatorname{Tot}}(X)=\bigoplus_{i \in \mathbb{Z} / 4} \bigoplus_{\mathcal{L} \in \operatorname{Pic}(X) / 2} \operatorname{PDiag}^{(i)}(X, \mathcal{L})
$$

the graded sub-semiring of $\operatorname{Symm}^{\text {Tot }}(X)$ generated by all symmetric pairs $\alpha(s ; \mathcal{L})$ for all global sections $s: \mathcal{O}_{X} \rightarrow \mathcal{L}$ of line bundles and by all their cones $d(\alpha(s ; \mathcal{L}))$. We call it the total subsemiring of pseudo-diagonal symmetric pairs.

We now give some examples.

Notation 6.7. Let $n \geqslant 0$. Consider $n+1$ line bundles $\mathcal{L}_{0}, \ldots, \mathcal{L}_{n} \in \operatorname{Pic}(X)$ and consider global sections $s_{j}: \mathcal{O}_{X} \rightarrow \mathcal{L}_{j}$ for $j=0, \ldots, n$. Consider the following pseudo-diagonal pair involving one symmetric pair and $n$ symmetric cones:

$$
\beta\left(s_{0}, \ldots, s_{n} ; \mathcal{L}_{0}, \ldots, \mathcal{L}_{n}\right):=\alpha\left(s_{0} ; \mathcal{L}_{0}\right) \star d \alpha\left(s_{1} ; \mathcal{L}_{1}\right) \star \cdots \star d \alpha\left(s_{n} ; \mathcal{L}_{n}\right) .
$$

This symmetric pair, sometimes only written $\beta\left(s_{0}, \ldots, s_{n}\right)$, defines an element of $\operatorname{PDiag}^{(n)}(X, \mathcal{L}) \subset$ $\operatorname{Symm}^{(n)}(X, \mathcal{L})$ where $\mathcal{L}:=\mathcal{L}_{0} \otimes \cdots \otimes \mathcal{L}_{n} \in \operatorname{Pic}(X) / 2$ since all factors contribute to a twist by $\mathcal{L}_{i}$ but only the last $n$ factors contribute to a shift by 1 . For $n=0$, we simply have $\beta\left(s_{0}\right)=\alpha\left(s_{0}\right)$. For instance, $\beta\left(1 ; \mathcal{O}_{X}\right)=\langle 1\rangle$.

Remark 6.8. By Proposition 6.5, the support $\operatorname{Supp}\left(\beta\left(s_{0}, \ldots, s_{n} ; \mathcal{L}_{0}, \ldots, \mathcal{L}_{n}\right)\right)$ of such a form is equal to $\operatorname{Supp}\left(\alpha\left(s_{0} ; \mathcal{L}_{0}\right)\right) \cap \bigcap_{i=1}^{n} \operatorname{Supp}\left(d \alpha\left(s_{i} ; \mathcal{L}_{i}\right)\right)=\bigcap_{i=1}^{n} Z\left(s_{i}\right)$. We consider its degeneracy locus in Proposition 6.11.

Proposition 6.9. Let $i \in \mathbb{Z} / 4$ and $\mathcal{L} \in \operatorname{Pic}(X) / 2$. Then, any element of the monoid of pseudodiagonal $\mathcal{L}$-twisted symmetric $i$-pairs $\operatorname{PDiag}^{(i)}(X, \mathcal{L})$, as defined in Definition 6.6, is an orthogonal sum of symmetric pairs $\beta\left(s_{0}, \ldots, s_{n} ; \mathcal{L}_{0}, \ldots, \mathcal{L}_{n}\right)$ as defined in Notation 6.7 , for various integers $n \equiv i$ modulo 4 and for various families of $n$ line bundles $\mathcal{L}_{0}, \ldots, \mathcal{L}_{n} \in \operatorname{Pic}(X)$ such that $\mathcal{L}_{0} \otimes \cdots \otimes \mathcal{L}_{n} \equiv \mathcal{L}$ modulo $2 \operatorname{Pic}(X)$.

Proof. Observe the following two properties:

(a) $-\alpha\left(s_{0} ; \mathcal{L}_{0}\right)=\alpha\left(-s_{0} ; \mathcal{L}_{0}\right)$ by definition of $-\alpha$, see Remark 2.6 ;

(b) $\alpha\left(s_{1} ; \mathcal{L}_{1}\right) \star \alpha\left(s_{2} ; \mathcal{L}_{2}\right)=\alpha\left(s_{1} \otimes s_{2} ; \mathcal{L}_{1} \otimes \mathcal{L}_{2}\right)$ by definition of the product.

Now, by construction, $\operatorname{PDiag}^{(i)}(X, \mathcal{L})$ is the $(i, \mathcal{L})$-graded part of $\operatorname{PDiag}^{\text {Tot }}(X)$ and the latter is the sub-semiring of $\operatorname{Symm}^{\text {Tot }}(X)$ generated by diagonal symmetric pairs $\alpha\left(s_{j} ; \mathcal{L}_{j}\right)$, see Definition 6.4 , and by their cones $d \alpha\left(s_{j} ; \mathcal{L}_{j}\right)$. So, a priori, an element of $\operatorname{PDiag}^{(i)}(X, \mathcal{L})$ is a sum of products of 


\section{Products of DEGENERATE QUADRATIC FORMS}

such pairs. Using commutativity and associativity up to signs (Lemma 6.3), we can regroup such a product as

$$
\pm \alpha\left(s_{1} ; \mathcal{L}_{1}\right) \star \cdots \star \alpha\left(s_{m} ; \mathcal{L}_{m}\right) \star d \alpha\left(s_{m+1} ; \mathcal{L}_{m+1}\right) \star \cdots \star d \alpha\left(s_{m+n} ; \mathcal{L}_{m+n}\right) .
$$

Using properties (a) and (b), we can regroup the $m$ factors with no ' $d$ ' into only one and we can even incorporate the possible sign into it. Such a product is a symmetric pair $\beta$ as in the statement.

Remark 6.10. This says that the symmetric forms $\beta\left(s_{0}, \ldots, s_{n} ; \mathcal{L}_{0}, \ldots, \mathcal{L}_{n}\right)$ of Notation 6.7 essentially describe all pseudo-diagonal forms, in the sense of Definition 6.6. These pseudo-diagonal forms constitute a natural generalization to arbitrary schemes of the usual diagonal forms over fields (where no ' $d$ ' intervenes). It would be interesting to know the answer to the following two open questions.

Question 1. When (i.e. over which schemes) is any symmetric space pseudo-diagonal?

Question 2. When is any symmetric space Witt-equivalent to a pseudo-diagonal space?

We only know that the answer to the weaker Question 2 is unaltered by passing from $X$ to $\mathbb{A}_{X}^{1}$ or to $\mathbb{P}_{X}^{n}$ when $X$ is regular (see $\S 7$ ). We now decide when such a pseudo-diagonal form $\beta\left(s_{0}, \ldots, s_{n}\right)$ is non-degenerate.

Proposition 6.11. We have $\operatorname{Deg} \operatorname{Loc}\left(\beta\left(s_{0}, \ldots, s_{n}\right)\right) \subset \bigcap_{i=0}^{n} Z\left(s_{i}\right)$.

Proof. By Proposition 6.5, $\operatorname{Supp}\left(\alpha\left(s_{0}\right)\right) \cap \operatorname{Supp}\left(d \alpha\left(s_{1}\right)\right) \cap \cdots \cap \operatorname{Supp}\left(d \alpha\left(s_{n}\right)\right)=Z\left(s_{1}\right) \cap \cdots \cap Z\left(s_{n}\right)$ whereas $\operatorname{DegLoc}\left(\alpha\left(s_{0}\right)\right) \cup \operatorname{DegLoc}\left(d \alpha\left(s_{1}\right)\right) \cup \cdots \cup \operatorname{DegLoc}\left(d \alpha\left(s_{n}\right)\right)=Z\left(s_{0}\right)$. So, by Definition 4.9, we obtain Cons $\left(\alpha\left(s_{0}\right), d \alpha\left(s_{1}\right), \ldots, d \alpha\left(s_{n}\right)\right)=\bigcap_{i=0}^{n} Z\left(s_{i}\right)$. Since the form $\beta\left(s_{0}, \ldots, s_{n}\right)$ is equal to the product $\alpha\left(s_{0}\right) \star d \alpha\left(s_{1}\right) \star \cdots \star d \alpha\left(s_{n}\right)$ by definition, the result now follows from Proposition 4.11.

Remark 6.12. Continuing Remarks 4.5 and 4.12, observe that equality holds in this statement. However, the above inclusion suffices to apply Proposition 3.4 and to obtain the following.

Corollary 6.13. For each $n \geqslant 0$, for each collection of $n+1$ line bundles $\mathcal{L}_{0}, \ldots, \mathcal{L}_{n}$ and for each family of global sections $s_{i} \in \Gamma\left(X, \mathcal{L}_{i}\right)$ such that $\bigcap_{i=0}^{n} Z\left(s_{i}\right)=\varnothing$, the pseudo-diagonal symmetric pair

$$
\beta\left(s_{0}, \ldots, s_{n}\right)=\alpha\left(s_{0}\right) \star d \alpha\left(s_{1}\right) \star \cdots \star d \alpha\left(s_{n}\right)
$$

is non-degenerate and hence defines a class in the Witt group $\mathrm{W}^{n}(X, \mathcal{L})$ where $\mathcal{L}=\mathcal{L}_{0} \otimes \cdots \otimes \mathcal{L}_{n}$.

Remark 6.14. Observe that Corollary 6.13 allows us to determine all non-degenerate pseudo-diagonal forms $\mathrm{MW}^{i}(X, \mathcal{L}) \cap \operatorname{PDiag}^{(i)}(X, \mathcal{L})$ by means of Proposition 6.9. They will be sums of pseudodiagonal spaces as in the corollary.

Remark 6.15. In Corollary 6.13, we do not say that $\alpha\left(s_{0}\right) \star \cdots \star \alpha\left(s_{n}\right)$ is non-degenerate since this is completely wrong. Indeed, the consanguinity of the forms $\alpha\left(s_{0}\right), \ldots, \alpha\left(s_{n}\right)$ is the union of the $Z\left(s_{i}\right)$ for $i=0, \ldots, n$ and so this product is non-degenerate only if every $s_{i}$ is an isomorphism. We do not consider $d \alpha\left(s_{0}\right) \star \cdots \star d \alpha\left(s_{n}\right)$ either, which is always non-degenerate, without assumption on the $Z\left(s_{i}\right)$, but is also always metabolic and hence of little interest for Witt groups, although they may define useful non-zero classes in Witt groups with support.

Let us draw the attention of the hurried reader to the asymmetry of the definition of $\beta\left(s_{0}, \ldots, s_{n}\right)$ in $s_{0}, \ldots, s_{n}$. The choice of having no $d$ only in front of $\alpha\left(s_{0}\right)$ is not so important up to Witt equivalence though. Indeed, suppose that $\bigcap_{i=0}^{n} Z\left(s_{i}\right)=\varnothing$, then it follows from commutativity and associativity of $\star$ (Lemma 6.3) and from the Leibniz formula (Corollary 5.3) that, up to signs, the Witt class of the symmetric space $\beta\left(s_{0}, \ldots, s_{n}\right)$ in $\mathrm{W}^{n}(X, \mathcal{L})$ does not depend on the order of the $s_{i}$, that is, for every permutation $\sigma$ of $\{0, \ldots, n\}$, we have

$$
\left[\beta\left(s_{0}, \ldots, s_{n} ; \mathcal{L}_{0}, \ldots, \mathcal{L}_{n}\right)\right]= \pm\left[\beta\left(s_{\sigma(0)}, \ldots, s_{\sigma(n)} ; \mathcal{L}_{\sigma(0)}, \ldots, \mathcal{L}_{\sigma(n)}\right)\right] .
$$




\section{P. BALMER}

Remark 6.16. It is clear that our graded semirings $\operatorname{Symm}^{\text {Tot }}(X), \operatorname{PDiag}^{\text {Tot }}(X)$, and so on, are functorial in $X$, in a contravariant way. Both definitions of $\alpha(s ; \mathcal{L})$ and of $\beta\left(s_{0}, \ldots, s_{n} ; \mathcal{L}_{0}, \ldots, \mathcal{L}_{n}\right)$ are natural in the obvious sense.

Proposition 6.17. For $n \geqslant 1$, under the condition that $\bigcap_{i=0}^{n} Z\left(s_{i}\right)=\varnothing$, the symmetric space $\beta\left(s_{0}, \ldots, s_{n} ; \mathcal{L}_{0}, \ldots, \mathcal{L}_{n}\right)$ is locally trivial in the Witt group, namely each point of $X$ has a neighborhood on which this space is metabolic.

Proof. We have by assumption a covering of $X$ by the complements of the $Z\left(s_{i}\right)$. On the first open $U_{0}:=X \backslash Z\left(s_{0}\right)$ the space becomes a product of a space $\left.\alpha\left(s_{0} ; \mathcal{L}_{0}\right)\right|_{U_{0}}$ with the metabolic space $\left.d\left(\alpha\left(s_{1} ; \mathcal{L}_{1}\right)\right) \star \cdots \star d\left(\alpha\left(s_{n} ; \mathcal{L}_{n}\right)\right)\right|_{U_{0}}$ and the product is therefore metabolic since $n \geqslant 1$. On the other open subsets $X \backslash Z\left(s_{i}\right)$ for $i=1, \ldots, n$ the object supporting the space is indeed zero since one of the factors is zero $\left.d \alpha\left(s_{i} ; \mathcal{L}_{i}\right)\right|_{X \backslash Z\left(s_{i}\right)}=0$. Hence, the result follows.

\section{Explicit examples over projective spaces}

In this section, $X$ is a scheme. Recall Conventions 0.2 and 0.3 .

Notation 7.1. Recall that $\mathbb{P}_{X}^{n}=\mathbb{P}_{\mathbb{Z}}^{n} \times_{\operatorname{Spec}(\mathbb{Z})} X$ where $\mathbb{P}_{\mathbb{Z}}^{n}=\operatorname{Proj}\left(\mathbb{Z}\left[T_{0}, \ldots, T_{n}\right]\right)$. For each $i=0, \ldots, n$, we also denote by $T_{i}$ the corresponding global section of $\mathcal{O}(1)$ over $\mathbb{P}_{\mathbb{Z}}^{n}$ and over $\mathbb{P}_{X}^{n}$ as well. We denote by

$$
Z_{i}:=Z\left(T_{i}\right) \subset \mathbb{P}_{X}^{n} \quad \text { and } \quad U_{i}:=\mathbb{P}_{X}^{n} \backslash Z_{i} \subset \mathbb{P}_{X}^{n}
$$

the closed subscheme $Z_{i} \simeq \mathbb{P}_{X}^{n-1}$ corresponding to ' $T_{i}=0$ ' and its open complement $U_{i} \simeq \mathbb{A}_{X}^{n}$. We shall also consider the closed subset

$$
Y:=Z_{1} \cap \cdots \cap Z_{n}=\left\{T_{1}=0, \ldots, T_{n}=0\right\} \subset U_{0} \subset \mathbb{P}_{X}^{n}
$$

corresponding to the point $[1: 0: \cdots: 0]$ of $\mathbb{P}^{n}$ and its open complement

$$
V:=\mathbb{P}_{X}^{n} \backslash Y \subset \mathbb{P}_{X}^{n}
$$

For simplicity we denote by the same letter $\pi$ all projection morphisms to $X$ :

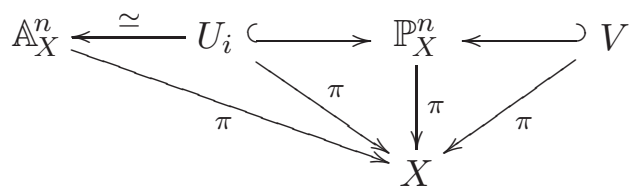

and even $\pi: \mathbb{P}_{X}^{n-1} \rightarrow X$. It is always clear from the context which projection is meant. For $n \geqslant 2$, we have a morphism over $X$

$$
\begin{aligned}
\eta: V & \longrightarrow \mathbb{P}_{X}^{n-1} \\
{\left[t_{0}: \cdots: t_{n}\right] } & \longmapsto\left[t_{1}: \cdots: t_{n}\right]
\end{aligned}
$$

which is obtained by base change to $X$ from the morphism over $\operatorname{Spec}(\mathbb{Z})$ described in the second line. For $n=1$ we make the convention that $\eta: V \rightarrow \mathbb{P}_{X}^{0}=X$ is the structure morphism $\pi$.

Remark 7.2. We adopt the following notation to drop unnecessary mentions of $\pi^{*}$.

(a) For $\mathcal{M} \in \operatorname{Pic}(X)$, we simply write $\mathrm{W}^{i}\left(\mathbb{P}_{X}^{n}, \mathcal{M}\right)$ to mean $\mathrm{W}^{i}\left(\mathbb{P}_{X}^{n}, \pi^{*} \mathcal{M}\right)$.

(b) For any class $w \in \mathrm{W}^{\operatorname{Tot}}\left(\mathbb{P}_{X}^{n}\right)$, the homomorphism $\mathrm{W}^{\operatorname{Tot}}(X) \longrightarrow \mathrm{W}^{\operatorname{Tot}}\left(\mathbb{P}_{X}^{n}\right)$ consisting in $\pi^{*}$ followed by multiplication by $w$ will simply be denoted by $\cdot w$ and will be called multiplication by $w$ (say, on the right). 


\section{Products of DEgENERATE QUADRATIC FORMS}

Definition 7.3. We apply the constructions of the previous sections.

(a) For any $i=0, \ldots, n$, following Definition 6.4 , we define the symmetric pair

$$
\alpha_{i}:=\alpha\left(T_{i} ; \mathcal{O}(1)\right) \in \operatorname{Symm}^{(0)}\left(\mathbb{P}_{X}^{n}, \mathcal{O}(1)\right) .
$$

(b) In Notation 6.7, we define the symmetric pair

$$
\begin{aligned}
\beta_{X}^{(n)} & :=\beta\left(T_{0}, \ldots, T_{n} ; \mathcal{O}(1), \ldots, \mathcal{O}(1)\right) \\
& =\alpha_{0} \star d \alpha_{1} \star \cdots \star d \alpha_{n} \in \operatorname{Symm}^{(n)}\left(\mathbb{P}_{X}^{n}, \mathcal{O}(n+1)\right) .
\end{aligned}
$$

Observing that $\bigcap_{i=0}^{n} Z_{i}=\varnothing$ we know from Corollary 6.13 that the above $\beta_{X}^{(n)}$ is non-degenerate and therefore defines a Witt class

$$
\left[\beta_{X}^{(n)}\right] \in \mathrm{W}^{n}\left(\mathbb{P}_{X}^{n}, \mathcal{O}(n+1)\right)
$$

(c) Using the short notation of Remark 7.2(b), we define a homomorphism

$$
\begin{aligned}
\left(1 \quad\left[\beta_{X}^{(n)}\right]\right): \mathrm{W}^{\operatorname{Tot}}(X) \oplus \mathrm{W}^{\operatorname{Tot}}(X) & \longrightarrow \mathrm{W}^{\operatorname{Tot}}\left(\mathbb{P}_{X}^{n}\right) \\
(\phi, \psi) & \longmapsto \pi^{*}(\phi)+\pi^{*}(\psi) \star\left[\beta_{X}^{(n)}\right] .
\end{aligned}
$$

Theorem 7.4. Let $X$ be a regular scheme and $n \geqslant 1$. The above homomorphism

$$
\left(1 \quad\left[\beta_{X}^{(n)}\right]\right): \mathrm{W}^{\operatorname{Tot}}(X) \oplus \mathrm{W}^{\operatorname{Tot}}(X) \longrightarrow \mathrm{W}^{\operatorname{Tot}}\left(\mathbb{P}_{X}^{n}\right)
$$

is an isomorphism. The ring structure is determined by the property that

$$
\left[\beta_{X}^{(n)}\right] \star\left[\beta_{X}^{(n)}\right]=0
$$

in $\mathrm{W}^{2 n}\left(\mathbb{P}_{X}^{n}\right)$ and by the fact that $\pi^{*}: \mathrm{W}^{\text {Tot }}(X) \rightarrow \mathrm{W}^{\text {Tot }}\left(\mathbb{P}_{X}^{n}\right)$ is a ring homomorphism.

Proof. We proceed by induction on $n \geqslant 1$, the case $n=1$ being treated at the very end of the proof. For any line bundle $\mathcal{L} \in \operatorname{Pic}\left(\mathbb{P}_{X}^{n}\right)$, we have a localization long exact sequence (see [Bal00, Theorems 6.2 and 6.8] or [Bal01b, Theorem 1.6])

$$
\cdots \longrightarrow \mathrm{W}_{Y}^{i}\left(\mathbb{P}_{X}^{n}, \mathcal{L}\right) \longrightarrow \mathrm{W}^{i}\left(\mathbb{P}_{X}^{n}, \mathcal{L}\right) \longrightarrow \mathrm{W}^{i}\left(V,\left.\mathcal{L}\right|_{V}\right) \stackrel{\partial_{\mathcal{L}}}{\longrightarrow} \mathrm{W}_{Y}^{i+1}\left(\mathbb{P}_{X}^{n}, \mathcal{L}\right) \longrightarrow \cdots
$$

where the connecting homomorphism $\partial_{\mathcal{L}}: \mathrm{W}^{i}\left(V,\left.\mathcal{L}\right|_{V}\right) \longrightarrow \mathrm{W}_{Y}^{i+1}\left(\mathbb{P}_{X}^{n}, \mathcal{L}\right)$ is induced by the cone construction, see $[$ Bal00, $\S 5]$. Before proceeding to a term-by-term analysis of (26), we recall, for those readers who might fear the loss of some twists in the following, that we have an isomorphism $\operatorname{Pic}(X) \oplus \mathbb{Z} \stackrel{\sim}{\longrightarrow} \operatorname{Pic}\left(\mathbb{P}_{X}^{n}\right)$ given by $(\mathcal{M}, m) \mapsto \pi^{*}(\mathcal{M})(m)=\pi^{*}(\mathcal{M}) \otimes \mathcal{O}(m)$. Also observe that the global section $T_{i}: \mathcal{O}_{\mathbb{P}_{X}^{n}} \rightarrow \mathcal{O}_{\mathbb{P}_{X}^{n}}(1)$ is an isomorphism outside $Z_{i}=Z\left(T_{i}\right)$, that is, on $U_{i} \simeq \mathbb{A}_{X}^{n}$. So, we have the following situation for Picard groups (written additively).

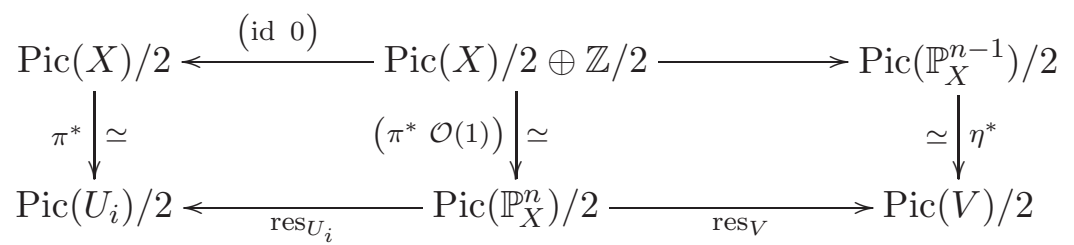

Note that, for $n=1$, the right-hand groups are isomorphic to $\operatorname{Pic}(X) / 2$ whereas for $n \geqslant 2$, we have $\operatorname{codim}_{\mathbb{P}_{X}^{n}}\left(\mathbb{P}_{X}^{n} \backslash V\right)=\operatorname{codim}_{\mathbb{P}_{X}^{n}}(Y)=n \geqslant 2$ so the restriction $\operatorname{res}_{V}: \operatorname{Pic}\left(\mathbb{P}_{X}^{n}\right) \rightarrow \operatorname{Pic}(V)$ is an isomorphism, see [Har77, $\S$ II.6]. Therefore, when $n \geqslant 2$, all morphisms in the right-hand square of (27) are isomorphisms.

Lemma 7.5. Consider the metabolic symmetric space $\gamma:=d \alpha_{1} \star \cdots \star d \alpha_{n}$ in $\operatorname{Symm}^{(n)}\left(\mathbb{P}_{X}^{n}, \mathcal{O}(n)\right)$. Let $\mathcal{M} \in \operatorname{Pic}(X) / 2$. Then, we have two isomorphisms

$$
\mathrm{W}^{i-n}(X, \mathcal{M}) \underset{\simeq}{\stackrel{\cdot[\gamma]}{\simeq}} \mathrm{W}_{Y}^{i}\left(\mathbb{P}_{X}^{n}, \mathcal{M}(n)\right)
$$




\section{P. BALMER}

and

$$
\mathrm{W}^{i-n}(X, \mathcal{M}) \underset{\sim\left[\beta_{X}^{(n)}\right]}{\simeq} \mathrm{W}_{Y}^{i}\left(\mathbb{P}_{X}^{n}, \mathcal{M}(n+1)\right)
$$

given by multiplication by the classes of $\gamma$ and of $\beta_{X}^{(n)}$, respectively.

Proof. First observe that $\operatorname{Supp}(\gamma)=\bigcap_{i=1}^{n} Z_{i}=Y$ and that therefore the first homomorphism is well-defined. Similarly we have $\operatorname{Supp}\left(\beta_{X}^{(n)}\right)=Y$, see Remark 6.8. Indeed, in Notation 6.7, we have $\gamma=\beta\left(1, T_{1}, \ldots, T_{n} ; \mathcal{O}, \mathcal{O}(1), \ldots, \mathcal{O}(1)\right)$.

On $\mathbb{A}_{X}^{n}:=\operatorname{Spec}\left(\mathbb{Z}\left[T_{1}^{\prime}, \ldots, T_{n}^{\prime}\right]\right) \times X$, consider the Koszul symmetric space $\kappa:=d \alpha_{1}^{\prime} \star \cdots \star$ $d \alpha_{n}^{\prime}$, where $\alpha_{i}^{\prime}:=\alpha\left(T_{i}^{\prime}, \mathcal{O}\right)$ following the notation of Definition 6.4. Gille has proved in [Gil03b, Theorem 9.3] that

$$
\mathrm{W}^{i-n}(X, \mathcal{M}) \stackrel{\cdot[\kappa]}{\longrightarrow} \mathrm{W}_{Y^{\prime}}\left(\mathbb{A}_{X}^{n}, \mathcal{M}\right) .
$$

is an isomorphism where $Y^{\prime}=\left\{T_{1}^{\prime}=0, \ldots, T_{n}^{\prime}=0\right\}$, at least in the case of $X$ affine and regular and of $\mathcal{M}=\mathcal{O}_{X}$. The global case is an immediate corollary of Gille's result by applying Mayer-Vietoris on the base $X$. Consider the morphisms

$$
\mathrm{W}^{i-n}(X, \mathcal{M}) \stackrel{\cdot[\kappa]}{\simeq} \mathrm{W}_{Y^{\prime}}^{i}\left(\mathbb{A}_{X}^{n}, \mathcal{M}\right) \underset{\simeq}{\simeq} \mathrm{W}_{Y}^{i}\left(U_{0}, \mathcal{M}\right) \stackrel{\operatorname{res}_{U_{0}}}{\simeq} \mathrm{W}_{Y}^{i}\left(\mathbb{P}_{X}^{n}, \mathcal{M}(m)\right) .
$$

The first is an isomorphism by the above result of Gille. The second is an isomorphism since $U_{0} \simeq \mathbb{A}_{X}^{n}$. The last isomorphism follows by Zariski excision (which is only a question of underlying categories, not of dualities), see [Bal01b, Corollary 2.3]. Note that $\left.\mathcal{O}(1)\right|_{U_{0}} \simeq \mathcal{O}_{U_{0}}$ and so the $m \in \mathbb{Z} / 2$ disappears on $U_{0}$.

We are left to show that the two following diagrams commute, one for $\gamma$ (with $m=n$ ) and one for $\beta_{X}^{(n)}$ (with $m=n+1$ ).

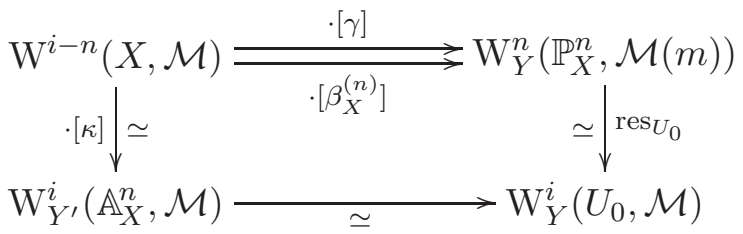

To see this, recall that the classical isomorphism $U_{0} \stackrel{\sim}{\rightarrow} \mathbb{A}_{X}^{n}$ corresponds to $T_{i}^{\prime} \mapsto T_{i} / T_{0}$ and that we use multiplication with $T_{0}$ to identify $\left.\mathcal{O}_{U_{0}} \simeq \mathcal{O}(1)\right|_{U_{0}}$. Therefore, this isomorphism $U_{0} \stackrel{\sim}{\rightarrow} \mathbb{A}_{X}^{n}$, which of course sends $Y$ to $Y^{\prime}$, also sends the symmetric pair $\alpha_{i}^{\prime}$ to $\alpha_{i}$ for $i=1, \ldots, n$. Via this isomorphism, we have a fortiori $[\kappa] \longmapsto\left[\left.\gamma\right|_{U_{0}}\right]=\left[\left.\beta_{X}^{(n)}\right|_{U_{0}}\right]$. This last equality follows from $\left.\left(\alpha_{0}\right)\right|_{U_{0}}=\langle 1\rangle$. This is the claimed commutativity and the lemma follows.

Lemma 7.6. Let $\mathcal{M} \in \operatorname{Pic}(X) / 2, m \in \mathbb{Z} / 2$. Suppose that $n \geqslant 2$. Then we have an isomorphism

$$
\eta^{*}: \mathrm{W}^{i}\left(\mathbb{P}_{X}^{n-1}, \mathcal{M}(m)\right) \stackrel{\sim}{\longrightarrow} \mathrm{W}^{i}(V, \mathcal{M}(m)) .
$$

For $n=1$, we simply have an isomorphism $\eta^{*}: \mathrm{W}^{i}(X, \mathcal{M}) \stackrel{\sim}{\rightarrow} \mathrm{W}^{i}(V, \mathcal{M})$.

Proof. The morphism $\eta: V \rightarrow \mathbb{P}_{X}^{n-1}$ is a vector bundle and the result follows by (generalized) homotopy invariance, see [Bal01b] or [Gil03a, Corollary 4.2].

Lemma 7.7. Suppose that $n \geqslant 2$. Recall $\gamma=d \alpha_{1} \star \cdots \star d \alpha_{n}$ from Lemma 7.5. The image of the class $\left[\beta_{X}^{(n-1)}\right] \in \mathrm{W}^{n-1}\left(\mathbb{P}_{X}^{n-1}, \mathcal{O}(n)\right)$ via the composition

$$
\mathrm{W}^{n-1}\left(\mathbb{P}_{X}^{n-1}, \mathcal{O}(n)\right) \stackrel{\eta^{*}}{\longrightarrow} \mathrm{W}^{n-1}(V, \mathcal{O}(n)) \stackrel{\partial}{\longrightarrow} \mathrm{W}_{Y}^{n}\left(\mathbb{P}_{X}^{n}, \mathcal{O}(n)\right)
$$

is given by $\partial\left(\eta^{*}\left[\beta_{X}^{(n-1)}\right]\right)= \pm[\gamma]$. Here, of course, $\partial=\partial_{\mathcal{O}(n)}$. 


\section{Products of Degenerate Quadratic Forms}

Proof. The definition of the connecting homomorphism $\partial$ is as follows. To compute $\partial\left(\left[\eta^{*}\left(\beta_{X}^{(n-1)}\right)\right]\right)$ we need to find a symmetric pair on $\mathbb{P}_{X}^{n}$ whose restriction to $V$ is the symmetric space $\eta^{*}\left(\beta_{X}^{(n-1)}\right)$ and then apply the symmetric cone construction $d$ to this 'lift'. See details in [Bal00, 5.16]. In formula, it means that we have

$$
\partial\left(\left[\eta^{*}\left(\beta_{X}^{(n-1)}\right)\right]\right)=\left[d\left(\alpha_{1} \star d \alpha_{2} \star \cdots \star d \alpha_{n}\right)\right]
$$

as soon as we observe that $\alpha_{1} \star d \alpha_{2} \star \cdots \star d \alpha_{n}$ is a symmetric pair on $\mathbb{P}_{X}^{n}$ whose restriction to $V$ is $\eta^{*}\left(\beta_{X}^{(n-1)}\right)$. The latter is obvious by definition of $\eta$, see $(25)$, and by definition of $\beta_{X}^{(n-1)}$, see Definition 7.3. Finally, we have

$$
\left[d\left(\alpha_{1} \star d \alpha_{2} \star \cdots \star d \alpha_{n}\right)\right] \stackrel{5.4}{=} \pm\left[d \alpha_{1} \star d \alpha_{2} \star \cdots \star d \alpha_{n}\right]= \pm[\gamma]
$$

which gives the lemma.

End of the proof of Theorem 7.4. For $n \geqslant 2$, consider the diagram

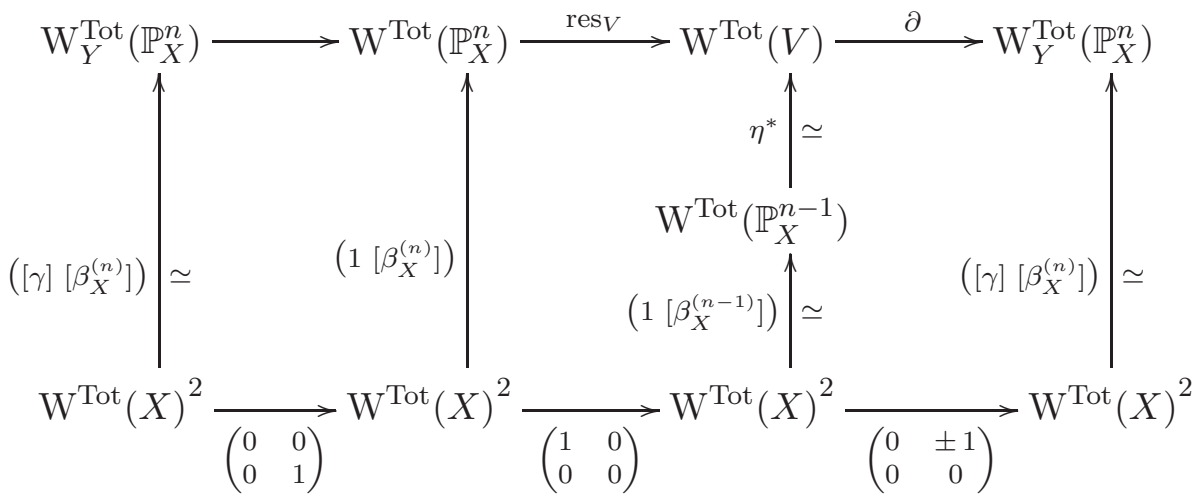

where $\mathrm{W}^{\text {Tot }}(X)^{2}=\mathrm{W}^{\text {Tot }}(X) \oplus \mathrm{W}^{\text {Tot }}(X)$ and where we of course use the notation $\mathrm{W}_{Y}^{\text {Tot }}\left(\mathbb{P}_{X}^{n}\right)$ to mean $\bigoplus_{i \in \mathbb{Z} / 4} \bigoplus_{\mathcal{L} \in \operatorname{Pic}\left(\mathbb{P}_{X}^{n}\right) / 2} \mathrm{~W}_{Y}^{i}\left(\mathbb{P}_{X}^{n}, \mathcal{L}\right)$.

Exactness of the first row is a compact form of the localization exact sequence (26). The second row is trivially exact.

The first (and last) vertical morphism is an isomorphism by Lemma 7.5. The vertical morphisms in the third column are isomorphisms by the induction hypothesis and by Lemma 7.6 for $\eta^{*}$.

To see commutativity of the first square it suffices to prove that $[\gamma]=0$ in $\mathrm{W}^{\text {Tot }}\left(\mathbb{P}_{X}^{n}\right)$, which is obvious since $\gamma=d \alpha_{1} \star \cdots \star d \alpha_{n}$ is a product of metabolic forms. To see commutativity of the second square, it suffices to see that $\operatorname{res}_{V}\left(\left[\beta_{X}^{(n)}\right]\right)=0$ in $\mathrm{W}^{\text {Tot }}(V)$ which is obvious since $\left[\beta_{X}^{(n)}\right]$ comes from $\mathrm{W}_{Y}^{\operatorname{Tot}}\left(\mathbb{P}_{X}^{n}\right)$, that is, $\beta_{X}^{(n)}$ is supported on $\bigcap_{i=1}^{n} Z_{i}=Y$ as we already checked in Remark 6.8 . To see commutativity of the third square it suffices to prove $\partial\left(\eta^{*}\left(\left[\beta_{X}^{(n-1)}\right]\right)\right)= \pm[\gamma]$, which is Lemma 7.7; indeed, the vanishing of the other component, i.e. $\partial \circ \eta^{*}=0$, is formal since $\eta^{*}$ factors via res $V$ : $\mathrm{W}^{\operatorname{Tot}}\left(\mathbb{P}_{X}^{n}\right) \rightarrow \mathrm{W}^{\operatorname{Tot}}(V)$ by commutativity of the second square.

We get the wanted isomorphism by the 5-Lemma, since diagram (28) is periodic. 


\section{P. BALMER}

For $n=1$, we have $V=U_{1}$ and hence $\alpha_{1}$ is non-degenerate on $V$ and defines a class in $\mathrm{W}^{0}\left(V,\left.\mathcal{O}(1)\right|_{V}\right)$. Consider the diagram

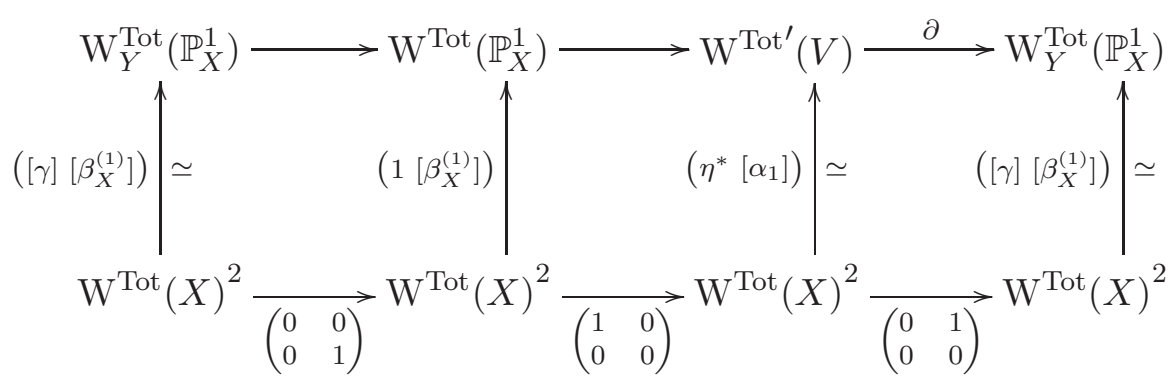

where we use $\mathrm{W}^{\operatorname{Tot}^{\prime}}(V)$ to mean $\bigoplus_{i \in \mathbb{Z} / 4} \bigoplus_{\mathcal{L} \in \operatorname{Pic}\left(\mathbb{P}_{X}^{1}\right) / 2} \mathrm{~W}^{i}\left(V,\left.\mathcal{L}\right|_{V}\right)$, which is not $\mathrm{W}^{\text {Tot }}(V)$ but rather two copies of it, since $\left.\mathcal{O}(1)\right|_{V} \cong \mathcal{O}_{V}$; see also (27). We need $\mathrm{W}^{\operatorname{Tot}^{\prime}}(V)$ because the first line of (29) is the sum over all shifts and all possible twists over $\mathbb{P}_{X}^{n}$ (not over $V$ !) of the localization exact sequence (26). Note also that the connecting homomorphism $\partial_{\mathcal{L}}$ of the localization exact sequence (26) depends on the 'ambient' category with duality, here $\left(\mathbb{D}^{\mathrm{b}}\left(\mathbb{P}_{X}^{n}\right), D_{\mathcal{L}}\right)^{(i)}$. So, although $\left[\alpha_{1}\right]=\langle 1\rangle$ if we identify $\mathrm{W}^{0}\left(V,\left.\mathcal{O}(1)\right|_{V}\right)$ with $\mathrm{W}^{0}(V)$, the connecting homomorphism which applies to $\alpha_{1}$ is the connecting homomorphism $\partial_{\mathcal{O}(1)}$ with respect to the twisted duality $D_{\mathcal{O}(1)}$. So, we get $\partial\left(\alpha_{1}\right)=$ $\left[d \alpha_{1}\right]=[\gamma]$ as wanted. The rest of the proof is as above: the diagram commutes and has two isomorphisms out of three by the previous lemmas.

To prove that $\left[\beta_{X}^{(n)}\right]^{2}=0$ observe that $\alpha_{0} \star d \alpha_{1} \star \cdots \star d \alpha_{n} \star \alpha_{0}$ is non-degenerate since $\alpha_{0}, d \alpha_{1}, \ldots, d \alpha_{n}$ have no consanguinity and since consanguinity does not change if we repeat some of the symmetric pairs (here $\alpha_{0}$ ) as can be verified directly on the definition, see Definition 4.9. Therefore, we are allowed to consider the Witt class of this space $\left[\alpha_{0} \star d \alpha_{1} \star \cdots \star d \alpha_{n} \star \alpha_{0}\right] \in \mathrm{W}^{n}\left(\mathbb{P}_{X}^{n}, \mathcal{O}(n)\right)$ and to make the following computation in $\mathrm{W}^{\text {Tot }}\left(\mathbb{P}_{X}^{n}\right)$ :

$$
\begin{aligned}
{\left[\beta_{X}^{(n)}\right]^{2} } & =\left[\alpha_{0} \star d \alpha_{1} \star \cdots \star d \alpha_{n} \star \alpha_{0} \star d \alpha_{1} \star \cdots \star d \alpha_{n}\right] \\
& =\left[\alpha_{0} \star d \alpha_{1} \star \cdots \star d \alpha_{n} \star \alpha_{0}\right] \star \underbrace{\left[d \alpha_{1} \star \cdots \star d \alpha_{n}\right]}_{=0}=0 .
\end{aligned}
$$

The latter class vanishes since the spaces $d \alpha_{i}$ are metabolic (and since $n \geqslant 1$ ).

Remark 7.8. In order to prove $\left(1\left[\beta_{X}^{n}\right]\right)$ an isomorphism, we can as well replace $\left[\beta_{X}^{(n)}\right]$ by its opposite $-\left[\beta_{X}^{(n)}\right]$. Therefore, any variation in the definitions leading to a sign change of $\beta_{X}^{(n)}$ does not really affect the presentation of the total Witt group of $\mathbb{P}_{X}^{n}$.

Remark 7.9. It is also immediate from the general considerations of Proposition 6.17 that the generator $\left[\beta_{X}^{(n)}\right]$ of $\mathrm{W}^{\mathrm{Tot}}\left(\mathbb{P}_{X}^{n}\right)$ is locally trivial on $\mathbb{P}_{X}^{n}$.

\section{ACKNOWLEDGEMENTS}

I would like to thank Stefan Gille, Bernhard Keller, Damian Mallaby, Fabien Morel, Damien Rößler and Charles Walter for various useful discussions. I am grateful to the referee for his very detailed reading and for his numerous suggestions.

\section{Appendix A. Recalling products, dualities and octahedra}

Definition A.1. Consider three triangulated categories $\mathcal{K}, \mathcal{L}$ and $\mathcal{M}$. A pairing of triangulated categories is a triple $(\otimes, \lambda, \rho)$ formed by a bifunctor

$$
\nabla: \mathcal{K} \times \mathcal{L} \longrightarrow \mathcal{M}
$$




\section{Products of Degenerate QUAdratic Forms}

which is exact in each variables with the natural isomorphisms

$$
\rho_{A, B}: A \otimes\left(T_{\mathcal{L}} B\right) \stackrel{\sim}{\rightarrow} T_{\mathcal{M}}(A \otimes B) \quad \text { and } \quad \lambda_{A, B}:\left(T_{\mathcal{K}} A\right) \otimes B \stackrel{\sim}{\rightarrow} T_{\mathcal{M}}(A \otimes B)
$$

expressing compatibility with translation for the exact functors $A \otimes-$ and $-\otimes B$. The following square is moreover assumed to be skew-commutative.

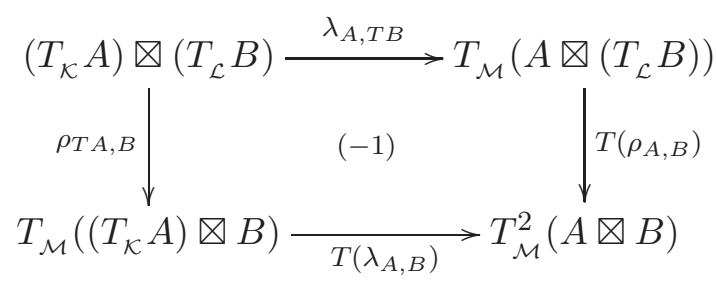

Definition A.2. A duality on a triangulated category $\mathcal{K}$ is a triple $(D, \delta, \varpi)$ where we have the following.

- $D: \mathcal{K}^{\mathrm{op}} \rightarrow \mathcal{K}$ is a \pm 1 -exact contravariant functor (the duality); exactness means, in particular, that $D \circ T=T^{-1} \circ D$.

- $\delta= \pm 1$ gives the exactness of $D$. So, 1-exact means exact and -1-exact means that distinguished triangles are sent to skew-distinguished triangles (those which are distinguished after changing the sign of the three morphisms).

- $\varpi: \operatorname{Id}_{\mathcal{K}} \rightarrow D \circ D$ is an isomorphism of functors, such that $D\left(\varpi_{A}\right) \circ \varpi_{D(A)}=\operatorname{id}_{D(A)}$ and $T\left(\varpi_{A}\right)=\varpi_{T(A)}$ for all $A \in \mathcal{K}$.

A triangulated category with duality is a quadruple $(\mathcal{K}, D, \delta, \varpi)$. See details in [Bal00].

Definition A.3. Given a triangulated category with duality $(\mathcal{K}, D, \delta, \varpi)$ and an integer $i \in \mathbb{Z}$, the ith shifted duality

$$
(D, \delta, \varpi)^{(i)}=\left(D^{(i)}, \delta^{(i)}, \varpi^{(i)}\right)
$$

on the same category $\mathcal{K}$ is defined by

$$
D^{(i)}:=T^{i} \circ D, \quad \delta^{(i)}:=(-1)^{i} \cdot \delta \quad \text { and } \quad \varpi^{(i)}:=(-1)^{i(i+1) / 2} \cdot \delta^{i} \cdot \varpi .
$$

It is easy to see that $(D, \delta, \varpi)^{(i+j)}=\left((D, \delta, \varpi)^{(i)}\right)^{(j)}$ for all $i, j \in \mathbb{Z}$.

Definition A.4 (Gille-Nenashev). Consider $\left(\mathcal{K}, D_{\mathcal{K}}, \delta_{\mathcal{K}}, \varpi_{\mathcal{K}}\right),\left(\mathcal{L}, D_{\mathcal{L}}, \delta_{\mathcal{L}}, \varpi_{\mathcal{L}}\right)$ and $\left(\mathcal{M}, D_{\mathcal{M}}, \delta_{\mathcal{M}}, \varpi_{\mathcal{M}}\right)$ three triangulated categories with duality. Following [GN03, Defenition 1.11], a pairing of triangulated categories with dualities between the three considered categories is a pair $(\mathbb{\nabla}, \mu)$ where:

- $\square: \mathcal{K} \times \mathcal{L} \longrightarrow \mathcal{M}$ is a pairing of triangulated categories (Definition A.1);

- $\mu$ is a natural isomorphism

$$
\mu_{A, B}: D_{\mathcal{K}} A \otimes D_{\mathcal{L}} B \stackrel{\sim}{\longrightarrow} D_{\mathcal{M}}(A \otimes B)
$$

such that the following two properties are satisfied.

(PD1) The following diagram commutes.

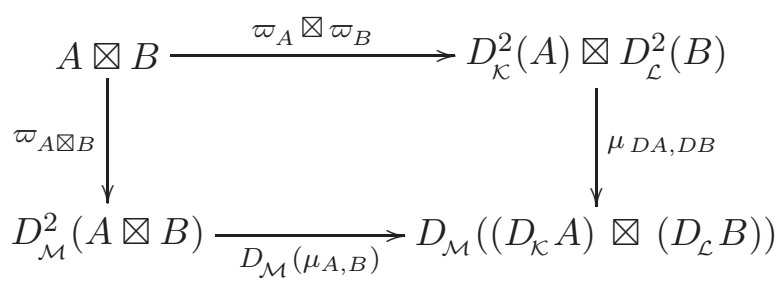




\section{P. BALMER}

(PD2) The following diagram commutes up to signs (given in the center).

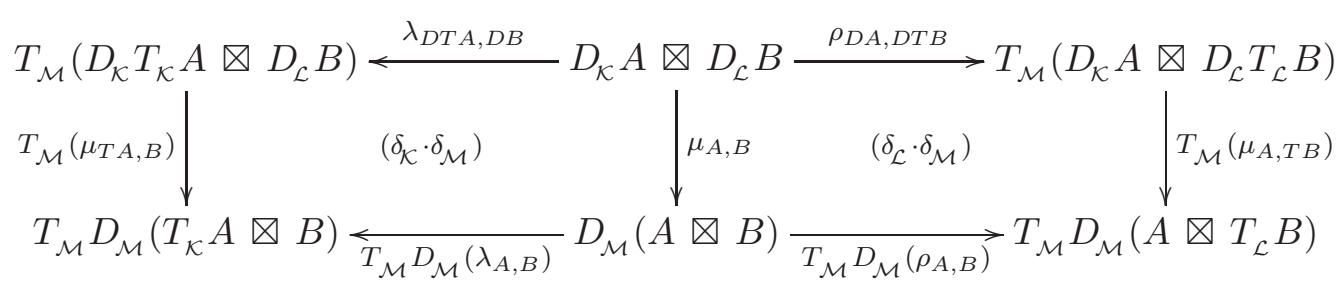

In the special case where $\mathcal{K}=\mathcal{L}=\mathcal{M}$, this gives the following.

Definition A.5. A triangulated category with product and duality, or in short a TPD-category is a triple $(\mathcal{K},(D, \delta, \varpi),(\otimes, \mu))$ where $(\mathcal{K}, D, \delta, \varpi)$ is a triangulated category with duality (Definition A.2) and $(\otimes, \mu)$ with

$$
\otimes: \mathcal{K} \times \mathcal{K} \rightarrow \mathcal{K}
$$

is a pairing compatible with the duality as in Definition A.4.

Definition A.6. Consider a pairing $(\otimes, \mu)$ of triangulated categories with duality $\otimes: \mathcal{K} \times \mathcal{L} \longrightarrow \mathcal{M}$

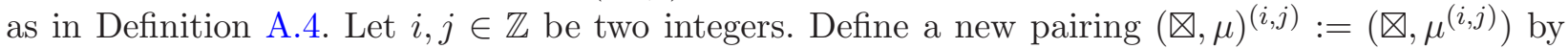
the formula

$$
\mu_{A, B}^{(i, j)}:=T_{\mathcal{M}}^{i+j}\left(\mu_{A, B}\right) \circ T_{\mathcal{M}}^{i}\left(\rho_{D A, D B}^{(j)}\right) \circ \lambda_{D A, D^{(j)} B}^{(i)}
$$

where $\lambda_{A, B}^{(i)}:\left(T_{\mathcal{K}}^{i} A\right) \otimes B \stackrel{\sim}{\rightarrow} T_{\mathcal{M}}^{i}(A \otimes B)$ and $\rho_{A, B}^{(j)}: A \otimes\left(T_{\mathcal{K}}^{j} B\right) \stackrel{\sim}{\rightarrow} T_{\mathcal{M}}^{j}(A \otimes B)$ are the obvious iterations of $\lambda$ and $\rho$. More explicitly,

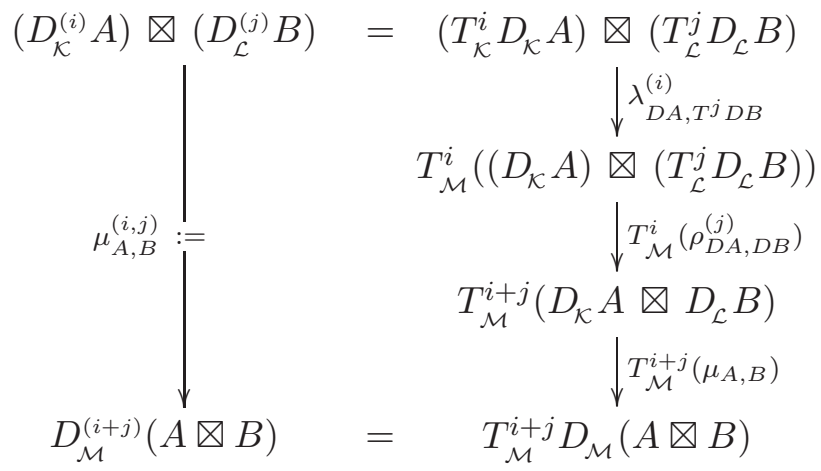

Proposition A.7. With notation of Definition A.6, the pairing $\nabla: \mathcal{K} \times \mathcal{L} \rightarrow \mathcal{M}$ is also compatible

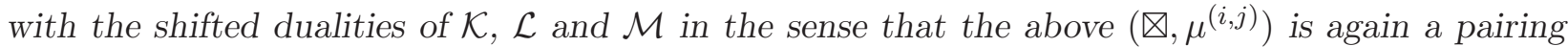
of triangulated categories with duality from the pair $\left(\mathcal{K}, D^{(i)}, \delta^{(i)}, \varpi^{(i)}\right),\left(\mathcal{L}, D^{(j)}, \delta^{(j)}, \varpi^{(j)}\right)$ and with values in $\left(\mathcal{M}, D^{(i+j)}, \delta^{(i+j)}, \varpi^{(i+j)}\right)$.

Proof. We do not give all of the details, but a pattern the cautious reader can follow.

First, we prove that $\mu^{(1,0)}$ turns $\otimes$ into a pairing of triangulated categories with duality between $\left(\mathcal{K}, D^{(1)}, \delta^{(1)}, \varpi^{(1)}\right) \times\left(\mathcal{L}, D^{(0)}, \delta^{(0)}, \varpi^{(0)}\right)$ and $\left(\mathcal{M}, D^{(1)}, \delta^{(1)}, \varpi^{(1)}\right)$.

To check the new (PD1), proceed as follows: first write it down; then replace $\mu^{(1,0)}$ by its definition (write this inside the diagram); then the central diagram is the juxtaposition of the old (PD1) and of the old (PD2) for $(D A, D B)$ instead of $(A, B)$; use also that $\varpi^{(1)}=(-\delta) \cdot \varpi$.

To check the new (PD 2), proceed as follows: first write it down; then replace $\mu^{(1,0)}$ by its definition (write this inside the diagram); then the left-hand diagram is the translation $T_{\mathcal{M}}$ of the left square of the old (PD2) plus the fact that $T D T=D$, whereas the right-hand diagram is obtained by the juxtaposition of the translation $T_{\mathcal{M}}$ of the right square of the old (PD2) with diagram (30).

The similar statement for $\mu^{(0,1)}$ can be established by following the mirror argument. Then, by induction, the statement holds for $\mu^{(i, 0)}$ and $\mu^{(0, j)}$. Finally, we use that $\mu^{(i, j)}=\left(\mu^{(0, j)}\right)^{(i, 0)}$. 


\section{Products of DEgENERATE QUADRATIC FORMS}

Remark A.8. Two words of caution about the definition of $\mu^{(i, j)}$ : there is no sign choice hidden in the definitions of $\lambda^{(i)}$ and $\rho^{(j)}$. They can be given explicitly as in [GN03, Remark 1.1]. There are sign choices in the definition of $\mu^{(i, j)}$ coming from the order in which we apply the natural isomorphism $\lambda, \rho$ and $\mu$. This roots back to the possible skew-commutativity of diagrams (30) and (PD 2), which roughly say that these natural isomorphisms only commute up to signs. With this in mind, there is not really a distinction between a left and a right product as in [GN03] but rather lots of choices for the order of stage appearance of $\lambda, \rho$ and $\mu$ in the definition of the natural isomorphism $\mu^{(i, j)}$, all choices giving the same result up to sign.

We now turn to the compatibility of product and triangulation. First, recall the following.

Definition A.9. In a triangulated category, an octahedron is a diagram as follows

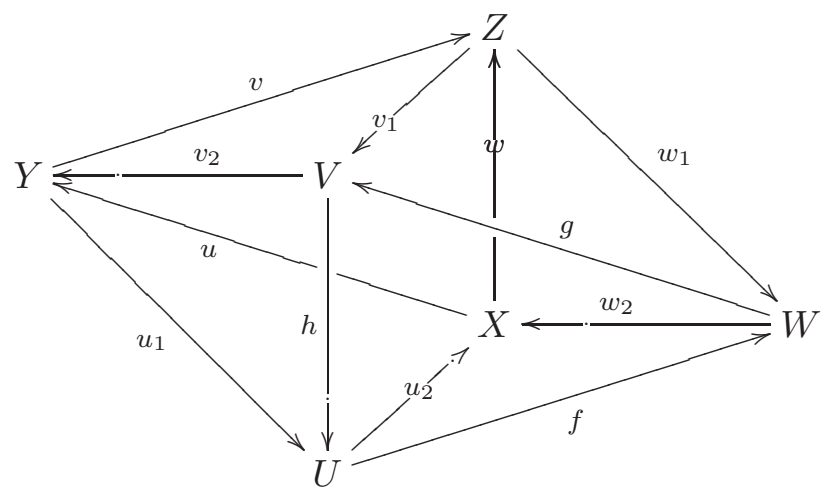

in which the four morphisms pictured with a broken arrow $\longrightarrow$ are of degree one; they are $u_{2}: U \rightarrow T X, v_{2}: V \rightarrow T Y, w_{2}: W \rightarrow T X$ and $h: V \rightarrow T U$. This octahedron is called distinguished if the following conditions hold:

(Oct1) the four triangles which can commute $(\nearrow \nwarrow)$ do commute;

(Oct2) the four triangles which can be distinguished (

(Oct3) both ways from $Y$ to $W$ coincide: $w_{1} v=f u_{1}$;

(Oct4) both ways from $W$ to $T(Y)$ coincide: $T(u) w_{2}=v_{2} g$.

If, moreover, to close the ring, the following two triangles containing the morphisms of (Oct3) and (Oct4) are distinguished:

(Oct5) $Y \stackrel{s}{\longrightarrow} W \stackrel{\left(\begin{array}{c}g \\ w_{2}\end{array}\right)}{\longrightarrow} V \oplus T X \stackrel{\left(v_{2}-T u\right)}{\longrightarrow} T Y$ where $s:=w_{1} v \stackrel{\text { Oct3 }}{=} f u_{1}$;

(Oct6) $Y \stackrel{\left(\begin{array}{c}u_{1} \\ v\end{array}\right)}{\longrightarrow} U \oplus Z \stackrel{\left(-f w_{1}\right)}{\longrightarrow} W \stackrel{t}{\longrightarrow} T Y$ where $t:=T(u) w_{2} \stackrel{\text { Oct4 }}{=} v_{2} g$;

then we say that (31) is a very distinguished octahedron. In this case, all morphisms extractable from octahedron (31) have an explicit distinguished triangle to live in.

In a triangulated category,

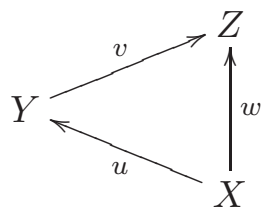




\section{P. BALMER}

the octahedron axiom (TR4), or composition axiom, asserts that any commutative triangle as above can be completed into a distinguished octahedron (31). The enriched octahedron axiom (TR4 ${ }^{+}$) asserts the same with a very distinguished octahedron. Our triangulated categories are assumed to satisfy $\left(\mathrm{TR} 4^{+}\right)$, as all known triangulated categories do. This enrichment is due to Beilinson et al. [BBD82].

Remark A.10. The following axiomatization of tensor triangulated categories comes from May [May01] and Keller and Neeman [KN02]. Consider a pairing of triangulated categories $\otimes: \mathcal{K} \times$ $\mathcal{L} \longrightarrow \mathcal{M}$ as in Definition A.1 and two distinguished triangles in $\mathcal{K}$ and $\mathcal{L}$, respectively:

$$
\begin{aligned}
& A \stackrel{a}{\longrightarrow} A^{\prime} \stackrel{a_{1}}{\longrightarrow} A^{\prime \prime} \stackrel{a_{2}}{\longrightarrow} T A, \\
& B \longrightarrow b_{1} B^{\prime} \stackrel{b_{1}}{\longrightarrow} B^{\prime \prime} \stackrel{b_{2}}{\longrightarrow} T B .
\end{aligned}
$$

Choose one morphism in each triangle, say $a$ and $b$, and write their product as

$$
a \otimes b=\left(\operatorname{id}_{A^{\prime}} \otimes b\right) \circ\left(a \otimes \operatorname{id}_{B}\right) .
$$

This produces an octahedron. Note, however, that the above product can also be decomposed as $a \otimes b=\left(a \otimes \operatorname{id}_{B^{\prime}}\right) \circ\left(\operatorname{id}_{A} \otimes b\right)$, yielding another octahedron. In good logic, since there are nine such pairs of morphisms, we can a priori produce 18 octahedra. As the reader would expect, there is some redundancy in this way of axiomatizing the relation between $\otimes$ and octahedra. Minimizing the redundancy is precisely the point of the following definition.

Definition A.11. We say that a pairing of triangulated categories $\nabla: \mathcal{K} \times \mathcal{L} \longrightarrow \mathcal{M}$ as in Definition A.1 is compatible with the octahedron axiom if for any two distinguished triangles (32) and (33) in $\mathcal{K}$ and $\mathcal{L}$, respectively, there exists an object $E$ in $\mathcal{M}$ and distinguished triangles in $\mathcal{M}$ :

$$
\begin{gathered}
A \otimes B \stackrel{a \otimes b}{\longrightarrow} A^{\prime} \otimes B^{\prime} \stackrel{c}{\longrightarrow} E \stackrel{d}{\longrightarrow} T(A \otimes B), \\
A^{\prime \prime} \otimes B \stackrel{e}{\longrightarrow} E \stackrel{f}{\longrightarrow} A^{\prime} \otimes B^{\prime \prime} \stackrel{\rho \circ\left(a_{1} \otimes b_{2}\right)}{\longrightarrow} T\left(A^{\prime \prime} \otimes B\right), \\
A \otimes B^{\prime \prime} \stackrel{g}{\longrightarrow} E \stackrel{h}{\longrightarrow} A^{\prime \prime} \otimes B^{\prime} \stackrel{\lambda \circ\left(a_{2} \otimes b_{1}\right)}{\longrightarrow} T\left(A \otimes B^{\prime \prime}\right),
\end{gathered}
$$

involving the same object $E$ and six morphisms $c, d, e, f, g$ and $h$, such that the following three octahedra are (very) distinguished.

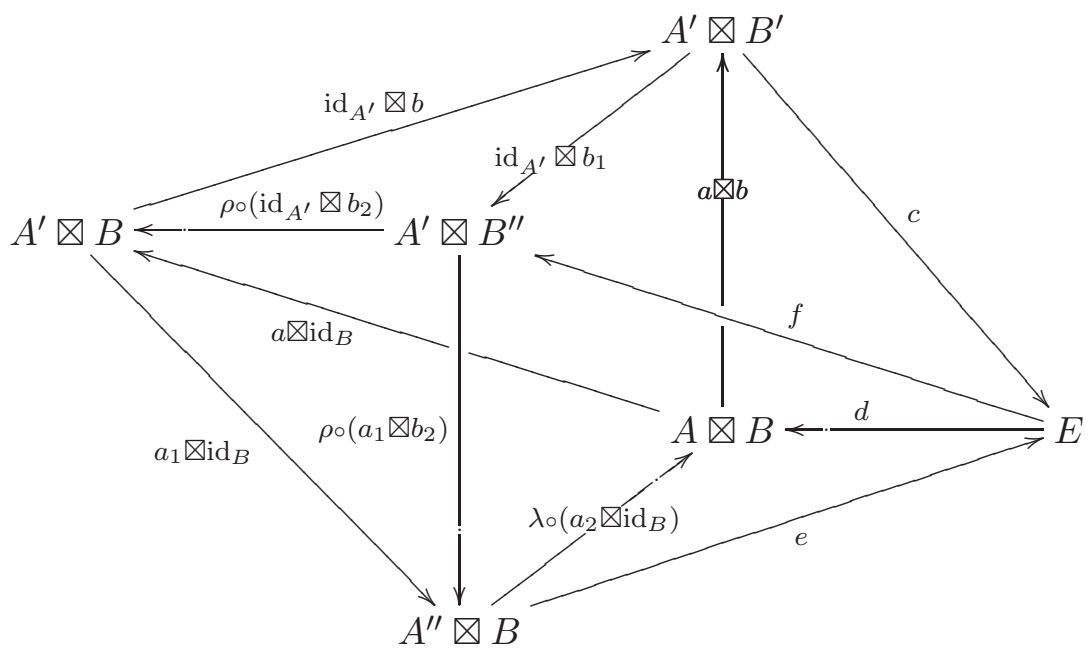



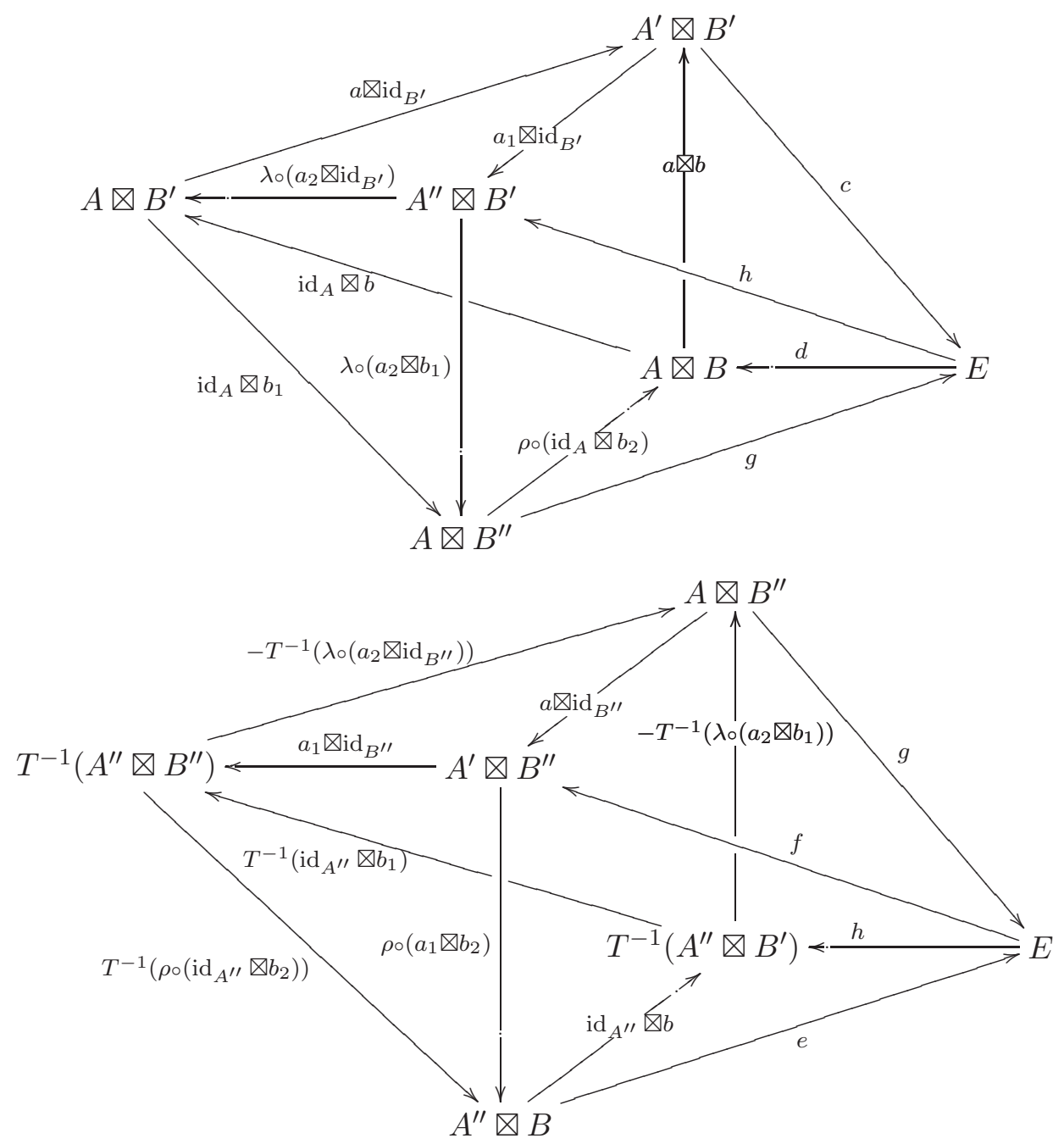

Remark A.12. The information encapsulated in the (very) distinction of those three octahedra is exactly equivalent to May's axiom (TC3') of [May01]. (Easier to check with May's (TC3') of [May01, Lemma 4.7].) For more on tensor triangulated categories, see Keller and Neeman [KN02]. Note that distinguished octahedra with objects exactly as above always exist without any extra compatibility axiom. The real point of Definition A.11 is that these three octahedra can be built with the same six morphisms $c, d, e, f, g$ and $h$, each of them staging in two different octahedra.

Remark A.13. We can always replace the object $E$ obtained in Definition A.11 via an isomorphism $\ell: E \stackrel{\sim}{\rightarrow} E^{\prime}$, changing accordingly the morphisms $c, d, e, f, g$ and $h$ having source or target equal to $E$, into the six morphisms

$$
\ell \circ c, \quad d \circ \ell^{-1}, \quad \ell \circ e, \quad f \circ \ell^{-1}, \quad \ell \circ g \text { and } h \circ \ell^{-1} .
$$

This allows us to choose one distinguished triangle among (34), (35) and (36). Moreover, once one of these triangles is chosen, say the second (35) (that is, if we want to keep $e$ and $f$ as they are), then we can still apply the above procedure a second time to modify the four other morphisms $c$, $d, g$ and $h$, but only with an automorphism $\ell: E \rightarrow E$ such that $\ell \circ e=e$ and $f \circ \ell^{-1}=f$. This is what we do in the proof of Theorem 5.2. The new octahedra are again (very) distinguished, since this property is preserved by isomorphism of octahedra. 


\section{Products of DEgenerate QUADRATIC FORMS}

\section{REFERENCES}

Ara80 J. Arason, Der Wittring projektiver Räume, Math. Ann. 253 (1980), 205-212.

Bal00 P. Balmer, Triangular Witt groups, Part I: The 12-term localization exact sequence, K-Theory 19 (2000), 311-363.

Bal01a P. Balmer, Triangular Witt groups, Part II: From usual to derived, Math. Z. 236 (2001), 351-382.

Bal01b P. Balmer, Witt cohomology, Mayer-Vietoris, homotopy invariance and the Gersten Conjecture, $K$-Theory 23 (2001), 15-30.

Bal05 P. Balmer, Witt groups, in Handbook of K-Theory (Springer, Berlin, 2005).

BBD82 A. Beilinson, J. Bernstein and P. Deligne, Faisceaux pervers, Astérisque 100 (1982).

Gil02 S. Gille, On Witt groups with support, Math. Ann. 322 (2002), 103-137.

Gil03a S. Gille, Homotopy invariance of coherent Witt groups, Math. Z. 244 (2003), 211-233.

Gil03b S. Gille, A transfer morphism for Witt groups, J. reine angew. Math. 564 (2003), 215-233.

GN03 S. Gille and A. Nenashev, Pairings in triangular Witt theory, J. Algebra 261 (2003), 292-309.

Har77 R. Hartshorne, Algebraic geometry, Graduate Texts in Mathematics, vol. 52 (Springer, Berlin, 1977).

KN02 B. Keller and A. Neeman, The connection between May's axioms for a triangulated tensor product and Happel's description of the derived category of the Quiver $D_{4}$, Doc. Math. 7 (2002), 535-560.

May01 P. May, The additivity of traces in triangulated categories, Adv. Math. 163 (2001), 34-73.

Nen04 A. Nenashev, On the Witt groups of projective bundles and split quadrics: geometric reasoning, Preprint (2004), available online at http://www.math.uiuc.edu/K-theory/0696/.

Ver96 J.-L. Verdier, Catégories dérivées des catégories abéliennes, Astérisque 239 (1996).

Wal03 C. Walter, Grothendieck-Witt groups of projective bundles, Preprint (2003), available online at http://www.math.uiuc.edu/K-theory/0644/.

Wei94 C. Weibel, An introduction to homological algebra (Cambridge University Press, Cambridge, 1994).

Paul Balmer balmer@math.ethz.ch http://www.math.ethz.ch/ balmer

D-Math, ETH Zentrum, 8092 Zürich, Switzerland 